\title{
Large-Scale Streamwise Vortices in Turbulent Channel Flow Induced by Active Wall Actuations
}

\author{
Weidan $\mathrm{Ni}^{1,2}$, Lipeng $\mathrm{Lu}^{1}$, Jian Fang ${ }^{1,2, \star}$, Charles Moulinec ${ }^{2}$, and Yufeng $\mathrm{Yao}^{3}$ \\ ${ }^{1}$ National Key Laboratory of Science and Technology on Aero-Engine Aero-Thermodynamics, School of Energy and \\ Power Engineering, Beihang University, Beijing, 100191, China \\ ${ }^{2}$ Scientific Computing Department, Science \& Technology Facilities Council Daresbury Laboratory, Warrington, WA4 \\ $4 A D, U K$ \\ ${ }^{3}$ Department of Engineering Design and Mathematics, University of the West of England, Bristol, BS16 1QY, UK \\ *Correspondence: jian.fang@stfc.ac.uk; Tel.: +4401925 603149
}

\begin{abstract}
Direct numerical simulations of turbulent flow in a plane channel using spanwise alternatively distributed strips (SADS) are performed to investigate the characteristics of large-scale streamwise vortices (LSSVs) induced by small-scale active wall actuations, and their role in suppressing flow separation. SADS control is obtained by alternatively applying out-of-phase control (OPC) and in-phase control (IPC) to the wall normal velocity component of the lower channel wall only, in the spanwise direction. Besides the unperturbed channel flow simulated as a reference, four controlled cases with 1, 2, 3 and 4 pairs of OPC/IPC strips are studied in the present paper at $M=0.2$ and $R e=6,000$, based on the bulk velocity and the channel half height $h$. The case with 2 pairs of strips is the most effective in terms of generating large-scale motions. It corresponds to a strip width of $\Delta z^{+}=264$ based on the friction velocity of the unperturbed case. The Reynolds shear stress peak value is located in the main stream of the channel at about $0.39 \mathrm{~h}$ from the lower channel wall. It is also found that the OPC (resp. IPC) strips suppress (resp. enhance) the coherent structures and that leads to the creation of a vertical shear layer, which is responsible for the LSSVs presence. They are in a statistically steady state and their cores are located between two neighbouring OPC and IPC strips. These motions contribute significantly to the momentum transport in the wall normal and spanwise directions showing potential for flow separation suppression.
\end{abstract}

Keywords: SADS Control, IPC/OPC, Channel Flow, DNS

\section{INTRODUCTION}

Boundary layer flow separation happens in many industrial applications, such as for road vehicles, airfoils and turbine blades, among many others. One significant factor related to boundary layer separation is that it increases drag. Taking a road vehicle as an example, flow separation along the car rear body results in an increase of the total drag hence leading to an increase in fuel consumption and $\mathrm{CO}_{2}$ emissions. About 2.1 billion barrels of oil per year (per 2003 figures) is consumed by ocean shipping worldwide and 1.5 billion barrels and 1.2 billion barrels of oil per year for airline and trucking, respectively [1]. As reviewed by Leschziner et al. [2], the fuel consumption especially in civil aviation, but also in road and shipping transport, are responsible for around 30 per cent of global $\mathrm{CO}_{2}$ emissions. Saving the fuel consumption by imposing flow control contributes to economy and environment. Leschziner et al. [2], split the drag control strategies into three categories: the delay of transition, the reduction of near-wall turbulence and the suppression of separation, in which the second one is 
mainly achieved by suppressing momentum transport in the boundary layer to reduce friction drag, while the third one aims to reduce the pressure drag by suppressing flow separation mainly via the enhancement of the momentum transport. Therefore, the overall effect of the control relies on a trade-off between friction and pressure drag. Passive vortex generators (VGs), which are made as arrays of ribs and grooves with sizes of the order of the boundary layer thickness $\delta$ and in alignment with the streamwise direction [3], are an example of the way of realising drag reduction by enhancing momentum transport. VGs boost the near-wall momentum transport process by generating large-scale streamwise vortices (LSSVs) and the flow separation is either suppressed or delayed. Both the pressure drag and the unsteadiness of the flow can then be reduced. Rao and Kariya [4] first reported that VGs with $H / \delta \leq 0.625$ (namely the ratio of the device height $H$ to the boundary layer thickness) have shown better performance than conventional VGs with $H / \delta \sim 1$; several researchers have confirmed that VGs with $0.1 \leq H / \delta \leq 0.5$ can indeed provide sufficient momentum transfer to control flow separation [5-8]. While this concept works well, it faces certain technical difficulties in practical applications such as design integration and manufacturing because of local shape change.

Besides vortex generators, it is also worthwhile looking into methods that can generate large-scale flow structures by acting on near-wall small-scale turbulence, such as alternatively distributed riblets [9] or simple wall roughness [10]. They offer the possibility to reduce both the friction drag in the attached flow region and the pressure drag in the separated flow area. Nugroho et al. [9] observe in their experiments that large-scale spanwise periodicity could be induced by applying converging-diverging riblet-type surface roughness in a zero-pressure-gradient turbulent boundary layer. The mean velocity can decrease or increase, depending on the converging or diverging local geometry feature, and this in turn results in a pronounced spanwise modification of the boundary layer thickness. In the spanwise direction, alternate exchange of low- and high-momentum pathways (denoted as LMPs and HMPs, respectively) is also observed by Mejia-Alvarez et al. [11,12] in their research about turbulent boundary layer flow over irregular complex roughness. Mejia-Alvarez et al. [11] suggest that these large-scale LMPs and HMPs spanning across the entire boundary layer thickness are somewhat different from the instantaneous low- and high-momentum regions (LMRs and HMRs, respectively), which are often identified in instantaneous realisations of wall turbulence [13-17]. These LMPs and HMPs could further provide preferred pathways for instantaneous large-scale flow motions. Willingham et al. [10] and Vermaas et al. [18] identify similar spanwise variation by imposing a regularly distributed spanwise alternating wall roughness which could induce significant lateral exchange of momentum. Mejia-Alvarez et al. [11,12,19] show that turbulence kinetic energy (TKE) and Reynolds shear stress (RSS) are both reduced within LMPs whereas intense TKE and RSS regions coincide with the spanwise boundaries of LMP. The counter-rotating vortices located between the LMP and HMP can lift low-momentum fluid upward to the outer part of the boundary layer. A 
similar distribution of TKE and RSS is observed by Nugroho et al. [9] who study a more organised topography configuration of converging-diverging riblet-type surface roughness. Hinze et al. [20, 21] analyse the secondary motions based on the turbulent production and dissipation by applying the usual boundary-layer approximations to the balance equations for TKE. They suggest that when the production is greater than the dissipation in a localized region, the turbulence-poor fluid will be transported into this region by the generated secondary flow, and in the meantime the turbulence-rich fluid will be flowing out of the region and vice versa. Mejia-Alvarez et al. $[11,12]$ argue that the surface skin friction spanwise variation induces a vertical shear layer, which could cause spanwise transfer of high- and low-momentum fluid and consequently lead to the generation of secondary flow of the scale of the boundary layer thickness. Willingham et al. [10] consider that LMPs can be referred to Prandtl's second kind of secondary flows. Townsend [22] suggests that large-scale secondary motions as previously mentioned could be sustained by transverse shear stress gradients imposed onto the boundary layer. Koeltzsch et al. [23] also apply the spanwise alternating pattern of converging-diverging riblets on the wall of a turbulent pipe flow and significant changes of mean velocity field in the near-wall are found. These control devices are much smaller than the vortex generators but their effectiveness seems very promising, although their control mechanism is still far from understood and the devices not fully optimised. Therefore, this work will further investigate the characteristics of LSSVs generated by this type of small-scale control devices to understand the underlying mechanisms as well as their ability to suppress or delay boundary layer flow separation.

In this paper, spanwise alternatively distributed strips (SADS) control, made of alternatively imposed out-of-phase control (OPC) and in-phase control (IPC) wall normal velocity actuations, is applied to a turbulent plane channel flow. The simulations are carried out using direct numerical simulation (DNS) at a Reynolds number of $R e=6,000$ (based on the bulk velocity and the half height of the channel $h$ ) and at a low Mach number of $M=0.2$. This paper aims to prove the effectiveness of the newly proposed SADS small-scale wall control strategy in terms of the induced large-scale flow motions and to find the optimized value of the width of the control strip. The effect of the strip width is characterised and discussed. Finally DNS data analysis is performed before drawing conclusions.

\section{METHODOLOGY}

The governing equations are described first, followed by the introduction of the numerical method. Finally the computational setup is presented focusing on the SADS control method adopted in this study.

\subsection{Governing Equations}


The three-dimensional unsteady compressible Navier-Stokes equations applied to a Newtonian fluid are solved numerically in a general and time-invariant system. They are written in a strong conservative form as

$$
\frac{\partial \boldsymbol{Q}}{\partial t}+\frac{\partial \boldsymbol{E}_{i}}{\partial x_{i}}-\frac{\partial \boldsymbol{F}_{i}}{\partial x_{i}}=\boldsymbol{S}
$$

where $\boldsymbol{Q}=[\rho, \rho u, \rho v, \rho w, E]^{\mathrm{T}}$ is the solution vector, $t$ the time and $\boldsymbol{S}$ gathers potential source terms. The primary variables are the density $\rho$, the velocity components $u, v$ and $w$ and the total energy $E$. In the present paper, the following nomenclature is adopted. The indices $i=1,2,3$ correspond to the streamwise $\left(x_{1}=x\right)$, wall-normal $\left(x_{2}=y\right)$ and spanwise $\left(x_{3}=\right.$ $z$ ) directions, respectively. The notation $x_{i}=\left(x_{1}, x_{2}, x_{3}\right)$ is used to represent the Cartesian coordinates $(x, y, z)$. The standard Einstein summation notation applies. The static temperature $T$ and static pressure $P$ are related to the density $\rho$ via an equation of state which reads for an ideal gas, $P=\rho T /\left(\gamma M^{2}\right)$, where $\gamma$ is the specific heat capacity ratio set as 1.4 for air.

The convection terms $\boldsymbol{E}_{i}$ and the diffusion terms $\boldsymbol{F}_{i}$ in Eq. (1) are respectively expressed as

$$
\boldsymbol{E}_{i}=\left[\begin{array}{c}
\rho u_{i} \\
\rho u u_{i}+\delta_{1 i} P \\
\rho v u_{i}+\delta_{2 i} P \\
\rho w u_{i}+\delta_{3 i} P \\
(E+P) u_{i}
\end{array}\right] \quad \text { and } \quad \boldsymbol{F}_{i}=\left[\begin{array}{c}
0 \\
\tau_{1 i} \\
\tau_{2 i} \\
\tau_{3 i} \\
b_{i}
\end{array}\right] \text {, }
$$

where $u_{i}=\left(u_{1}, u_{2}, u_{3}\right)=(u, v, w)$ corresponds to the streamwise, wall-normal and spanwise velocity components, respectively. $\delta_{i j}$ stands for the Kronecker delta.

The total energy $E$ is expressed as

$$
E=\frac{1}{2}\left(\rho u_{i} u_{i}\right)+\frac{\rho T}{\gamma(\gamma-1) M^{2}} .
$$

The stress tensor $\tau_{i j}$ and the heat flux vector $b_{i}$ components are expressed as

$$
\tau_{i j}=\frac{\mu}{R e}\left(\frac{\partial u_{i}}{\partial x_{j}}+\frac{\partial u_{j}}{\partial x_{i}}-\frac{2}{3} \delta_{i j} \frac{\partial u_{k}}{\partial x_{k}}\right)
$$

and

$$
b_{i}=u_{j} \tau_{i j}+\frac{\mu}{\operatorname{PrRe}(\gamma-1) M^{2}} \frac{\partial T}{\partial x_{i}}
$$

where the Prandtl number $P r$ is defined in the following.

The dynamic viscosity coefficient $\mu$ is calculated as a function of temperature via the Sutherland law as:

$$
\mu=T^{1.5} \frac{T_{S} / T_{r e f}+1}{T+T_{S} / T_{r e f}}
$$

where $T_{\text {ref }}$ is the reference wall temperature $T_{r e f}=298.15 \mathrm{~K}$ and $T_{S}$ is the Sutherland constant temperature given as $T_{S}=110.4 K$ 
The source term $\boldsymbol{S}$ in the right-hand side of Eq. (1) will be given its specific form for channel flows in Subsection 2.3.

The Navier-Stokes equations are non-dimensionalised by the bulk density $\rho_{\text {bulk }}=\frac{\int \rho d V}{V}$, the bulk velocity $u_{\text {bulk }}=$ $\frac{\int \rho u d V}{\rho_{\text {bulk }} V}$, the reference temperature at the wall $T_{r e f}$, the reference dynamic viscosity $\mu_{\text {ref }}$ (namely the viscosity at the wall $\mu_{w}$ and calculated by Eq. (6) with $T=T_{r e f}$ ) and the half height of the channel, where $V$ stands for the volume of the computational domain calculated as $V=L_{x} \times L_{y} \times L_{z} . L_{x}, L_{y}$ and $L_{z}$ represent its length, height and width, respectively. The resulting dimensionless parameters are the Reynolds number $R e=\rho_{\text {bulk }} u_{\text {bulk }} h / \mu_{\text {ref }}$ and the Mach number $M=$ $u_{b u l k} / \sqrt{\gamma R T_{r e f}}$. The constant Prandtl number $\operatorname{Pr}=\mu C_{p} / k=0.72$ is used, where $C_{p}=\gamma R /(\gamma-1)$ is the specific heat capacity of a gas at constant pressure and $k$ is the thermal conductivity. $R$ is the gas constant, which is set to $R=$ $287.1 \mathrm{~J} /(\mathrm{kg} \cdot \mathrm{K})$.

\subsection{Numerical Method}

An in-house DNS code is used to solve the system written in Eq. (1). The Advanced flow Simulator for Turbulence Research (ASTR) code has been previously applied to DNS [24,25] and large-eddy simulation [26]. The Navier-Stokes equations are transformed using the Cartesian coordinate system in a computational domain and solved by high-order finite difference techniques. All the spatial derivatives are approximated using Lele's classic $6^{\text {th }}$-order compact central scheme [27].

The $2^{\text {nd }}$-order derivatives of the diffusion term are computed by applying twice the $1^{\text {st }}$-order differential operator. This approach is more computationally efficient than directly differencing the second-order derivatives [28], although the latter method could be numerically more stable. To remove small-scale wiggles due to aliasing errors resulting from the discrete evaluation of the nonlinear convection terms, a $10^{\text {th }}$-order compact filter is applied in order to limit the filtering impact at high wave numbers [29]. After all the spatial terms are solved, the $3^{\text {rd }}$-order total variation diminishing Runge-Kutta method [30] is used for time integration.

\subsection{Computational Setup}

A DNS of an unperturbed plane channel flow between two infinite plates is first conducted as the baseline case. This case is denoted as Case NC. All the settings are kept the same as those of Case NC for all the control cases, except for the velocity boundary condition at the lower wall where the control strategy is imposed. The Reynolds number is set to Re= 6,000, which corresponds to $R e_{\tau}=336$ based on the wall friction velocity $u_{\tau}$, where $u_{\tau}$ is defined as $u_{\tau}=\sqrt{\frac{\tau_{w}}{\rho_{w}}}$ and $\tau_{w}$ is the 
wall shear stress expressed as $\tau_{w}=\left.\mu_{w} \frac{\partial u}{\partial y}\right|_{w} \cdot \mu_{w}$ and $\rho_{w}$ are the dynamic viscosity coefficient and density at the wall, respectively; " $I_{w}$ " stands for the wall value. The Mach number is set to $M=0.2$. The size of the computational domain is $L_{x} \times L_{y} \times L_{z}=2 \pi h \times 2 h \times \pi h$ and the mesh is made of $392 \times 192 \times 256$ grid points in the $x$-, $y$ - and $z$-directions respectively. The mesh is uniformly distributed in the streamwise and spanwise directions and refined towards the walls in the wall-normal direction. The mesh resolution of a fully developed channel flow without SADS control (i.e. Case NC) matches the requirements for DNS of wall turbulence proposed by Sagaut [31]. The simulation settings for Case NC are summarised in TABLE 1 . All the superscripts "+" mean that the variables are non-dimensionalised by the wall viscous length scale $l_{\tau}$, which is calculated by the wall units of Case NC, expressed as $l_{\tau}=\frac{\mu_{w}}{\rho_{w} u_{\tau}}$. $\Delta t$ is the time step of the computation while $\Delta t^{+}$is the non-dimensional time step normalised by the viscous time scale $t_{\tau}=\frac{l_{\tau}}{u_{\tau}}$. The non-slip boundary condition with a fixed wall temperature $T_{w}=1$ is applied at both top and bottom walls, while periodic boundary conditions are prescribed in the streamwise and spanwise directions respectively.

TABLE 1. Settings for the simulation Case NC.

\begin{tabular}{cccccccccccc}
\hline \hline Case & $R e_{\tau}$ & $\Delta x$ & $\Delta x^{+}$ & $\Delta y_{1}$ & $\Delta y_{1}^{+}$ & $\Delta y_{\max }$ & $\Delta y_{\max }^{+}$ & $\Delta z$ & $\Delta z^{+}$ & $\Delta t \times 10^{4}$ & $\Delta t^{+} \times 10^{2}$ \\
\hline NC & 336 & 0.0160 & 5.39 & 0.00192 & 0.647 & 0.0203 & 6.83 & 0.0123 & 4.13 & 7.00 & 1.38 \\
\hline \hline
\end{tabular}

For all the cases studied the flow is driven by a streamwise body force based on the mass flow rate derived from Case NC. The body force is uniform and unsteady. It is used to compute the source term $\boldsymbol{S}$ of Eq. (1) as,

$$
\boldsymbol{S}=\left[\begin{array}{c}
0 \\
F_{x} \\
0 \\
0 \\
F_{x} u_{b u l k}
\end{array}\right] .
$$

$F_{x}$ is the streamwise body force. Following Lenormand et al. [32], the body force $F_{x}$ is calculated as

$$
F_{x}^{n+1}=F_{x}^{n}-\frac{\Delta t}{L_{y} L_{z}}\left[2\left(Q^{n+1}-Q_{0}\right)+0.2\left(Q^{n}-Q_{0}\right)\right]
$$

where $Q_{0}, Q^{n}, Q^{n+1}$ are, respectively, the initial mass flow rate (which remains constant), the mass flow rate at time step $n$ and the mass flow rate at time step $n+1$, given by a first-order predictor as

$$
Q^{n+1}=Q^{n}+\Delta t\left(L_{y} L_{z} F_{x}^{n}-f_{x}\right)
$$

$f_{x}$ is the averaged streamwise wall shear stress, calculated as

$$
f_{x}=\frac{1}{L_{x}} \int\left(\left.\mu \frac{\partial u}{\partial y}\right|_{y=0}-\left.\mu \frac{\partial u}{\partial y}\right|_{y=2 h}\right) d x d z
$$

The flow field is initialised by a laminar Poiseuille profile with super-imposed stochastic disturbances. It is expressed as 


$$
\left[\begin{array}{c}
\rho \\
u \\
v \\
w \\
T
\end{array}\right]=\left[\begin{array}{c}
\rho_{r e f} \\
1.5 u_{r e f}\left[1-(y / h-1)^{2}\right](1+\varphi) \\
0 \\
0 \\
T_{r e f} \frac{\gamma-1}{3} \operatorname{PrM}^{2} 1.5^{2}\left[1-(y / h-1)^{4}\right]
\end{array}\right],
$$

The stochastic disturbance $\varphi$ is in the range of $[-0.3,0.3]$.

After the flow reaches a fully developed turbulent state, SADS control is imposed onto the bottom wall only. The distribution of the 4 SADS control configurations is presented in Fig. 1(a). The upper channel wall is kept as a smooth nonslip wall whereas the lower channel wall undergoes alternated out-of-phase control and in-phase control actuations in the spanwise direction. The active wall-normal velocities at the lower channel wall imposed by OPC and IPC are respectively given by

$$
v_{w}(x, z)=-A_{O P C} v\left(x, y_{d t c}, z\right)
$$

and

$$
v_{w}(x, z)=+A_{I P C} v\left(x, y_{d t c}, z\right)
$$

$v_{w}(x, z)$ is the wall-normal velocity at the lower wall and $y_{d t c}$ is the non-dimensionalised distance from the wall, corresponding to a value of $y_{d t c}^{+} \approx 11$. This value, which is calculated with the wall values of Case NC, is close to the optimised value for drag reduction obtained by Choi et al. [33]. The other two velocity components at the wall are kept to zero. The coefficients $A_{O P C}$ and $A_{I P C}$ are two parameters controlling the amplitude of the wall velocities. They are both set to 0.5 in the present study, as this value helps keep the computation stable. Choi et al. [33] conducted a channel flow study with wall-normal velocities (Eq. (12), $A_{O P C}=1.0$ ) imposed on both the upper and lower walls, to explore possible drag reduction by suppressing sweep and ejection motions. They found that a substantial skin friction reduction of about $25 \%$ on each wall is achieved at $y_{d t c}^{+} \approx 10$. It was also demonstrated in a previous study [34] that applying IPC only induces significant skin friction increase and turbulence enhancement. Therefore, it is expected that the implementation of SADS control will generate alternatively high and low skin friction regions, creating a vertical shear layer due to the spanwise variation of the surface drag forces [11,12]. The principle of the adopted control method with two pairs of OPC/IPC strips is presented in Fig. 1(b). The instantaneous wall-normal velocity field is shown in Fig. 1 (c). A strong velocity normal to the wall is observed at the surface of the IPC strips due to the wall turbulence enhancement at the detected plane.

Four cases with different widths of OPC/IPC strips are studied and the settings are shown in TABLE 2. 
(a)

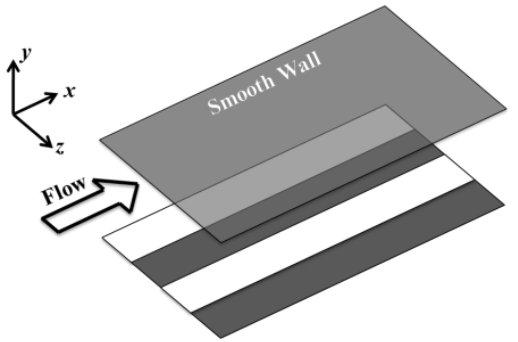

(b)

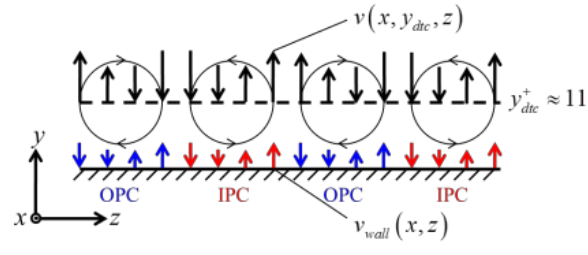

(c)

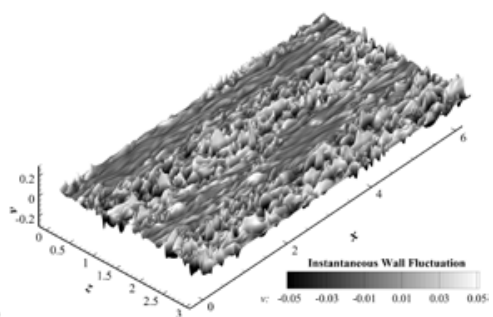

Fig. 1 Sketch of the topography configuration using two pairs of control strips (a). The principle of the control method is presented in (b). The instantaneous wall velocity resulting from the application of the control method is shown in (c). The lower wall of the channel, where OPC and IPC are applied, are highlighted by white and dark grey strips respectively in (a). The black and coloured arrows represent the wall-normal velocity at the detected plane and the wall respectively in (b).

TABLE 2. Details of the baseline and the four controlled cases

\begin{tabular}{cccc}
\hline \hline \multirow{2}{*}{ Case } & \multirow{2}{*}{ \#Strips } & \multicolumn{2}{c}{ Strip Width ${ }^{\mathrm{a}}$} \\
& & Based on $h$ & Based on $l_{\tau}$ \\
\hline NC & \multicolumn{2}{c}{ Baseline case without control } \\
Nstrip2 & 2 & $\pi / 2$ & 528 \\
Nstrip4 & 4 & $\pi / 4$ & 264 \\
Nstrip6 & 6 & $\pi / 6$ & 176 \\
Nstrip8 & 8 & $\pi / 8$ & 132 \\
\hline \hline
\end{tabular}

${ }^{a}$ For a given controlled case, the OPC and IPC strip widths are the same.

Considering Case NC, after the initial transient period, the flow becomes fully turbulent and reaches a statistically steady-state after about 110 time units. SADS control is then applied to the bottom channel wall to simulate Case Nstripn $(\mathrm{n}=$ $2,4,6,8)$. After the imposition of SADS control, the flow undergoes another transient period of around 240 time units before reaching a statistically steady state. For all the cases, the data samples are collected for about 205 time units before being post-processed and analysed.

In the present paper, ${ }^{-}$stands for the time-averaging operator and \langle\rangle for the space-averaging operator, i.e. \langle\rangle$_{x},\langle\rangle_{z}$ and \langle\rangle$_{x z}$ are used for the streamwise, spanwise and both streamwise and spanwise averaged variables, respectively. The fluctuations from each averaging operator are defined as $g^{\prime}=g-\bar{g}, g_{\langle x\rangle}=g-\langle g\rangle_{x}, g_{\langle z\rangle}=g-\langle g\rangle_{z}$ and $g_{\langle x z\rangle}=g-$ $\langle g\rangle_{x z}$ where $g$ can be any variable. The averaging operators can also be combined since they are all linear operators, i.e. $\langle\bar{g}\rangle_{x z}$ and $g^{\prime}{ }_{\langle x z\rangle}$ might be used, for instance.

\section{RESULTS AND DISCUSSION}

In Section 3.1, the results are primarily validated by comparing the baseline Case NC with the incompressible DNS database of Moser et al. [35]. Then, the turbulent coherent structures visualised by iso-surfaces of the swirling strength are presented for all the cases in Section 3.2 to intuitively illustrate the impact of SADS control on the turbulence intensity. The 
mean flow field data are analysed in Section 3.3 to study the influence of the width of the control strips on the mean statistics, including the skin friction coefficient $C_{f}$, the velocity profiles and the distribution of the mean streamwise velocity in the $y$ - $z$ plane. In Section 3.4, the turbulence kinetic energy and Reynolds shear stress are analysed in details. This is followed by the visualisation of the instantaneous flow field in Section 3.5 to further analyse Case Nstrip4 as it exhibits the maximum control effect among the four controlled cases studied.

\subsection{Validation}

The mean velocity profile handled by the Van Driest transformation in the inner scaling is validated by comparing Case NC (See TABLE 2) with the incompressible DNS data of Moser et al. [35] at a Reynolds number based on the friction velocity, of 395, as well as with the incompressible law of the wall (see Fig. 2). A good agreement in both the linear sublayer and the log-law layer is obtained by the present DNS.

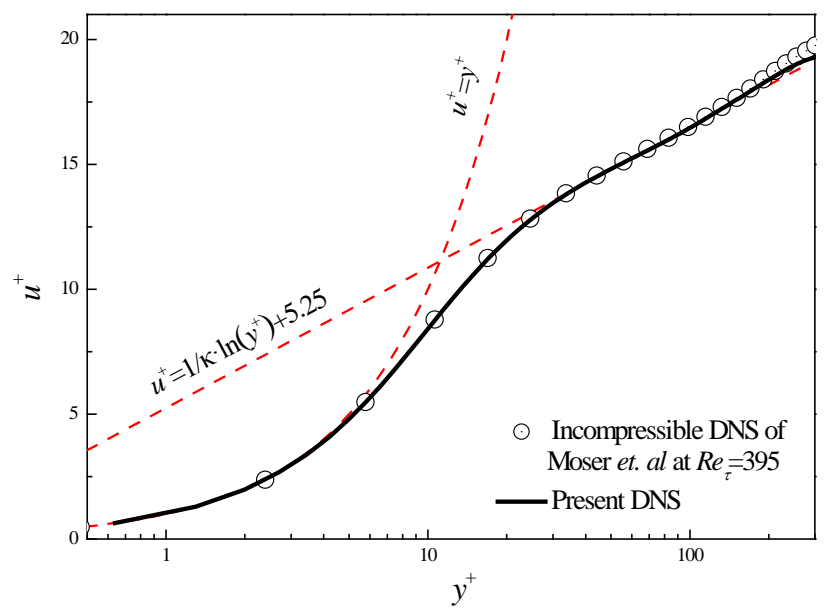

Fig. 2 Mean velocity profile in the inner scaling. The red dashed lines are for the incompressible law of the wall with a von Kármán constant of $\kappa=0.41$.

The Reynolds stress components of Case NC are also compared to the incompressible DNS data [35] in Fig. 3 and a general good agreement is achieved both in the near-wall region and the central part of the channel. There could be two reasons for the deviation of the present Case NC from the incompressible DNS of KMM database [35]. The first one is the compressibility effect. Even though the Mach number of the present simulation is within the incompressible range, the variety of density still has observable effect on the mean statistics. It can be seen from Fig. 3 (a) that, considering the change of density, the streamwise component of Reynolds stress is recalculated by $\left\langle u^{\prime} u^{\prime}\right\rangle_{\rho}=\left\langle\overline{u_{\langle x z\rangle}^{\prime} u_{\langle x z\rangle}^{\prime}}\right\rangle_{x z}\langle\bar{\rho}\rangle_{x z} /\langle\bar{\rho}\rangle_{x z, w}$ and the agreement of the peak of $\left\langle u^{\prime} u^{\prime}\right\rangle_{\rho}$ is improved compared with the one without taking account of density change. The other reason could be that the Reynolds number of the present study is different from the incompressible DNS of Moser et al. [35]. 
The friction Reynolds number of the present research is 340 whereas the results from Moser et al. [35] are simulated in a flow of $R e_{\tau}=395$, as the profile of Reynolds stress clearly shows a Reynolds number dependence in their DNS.
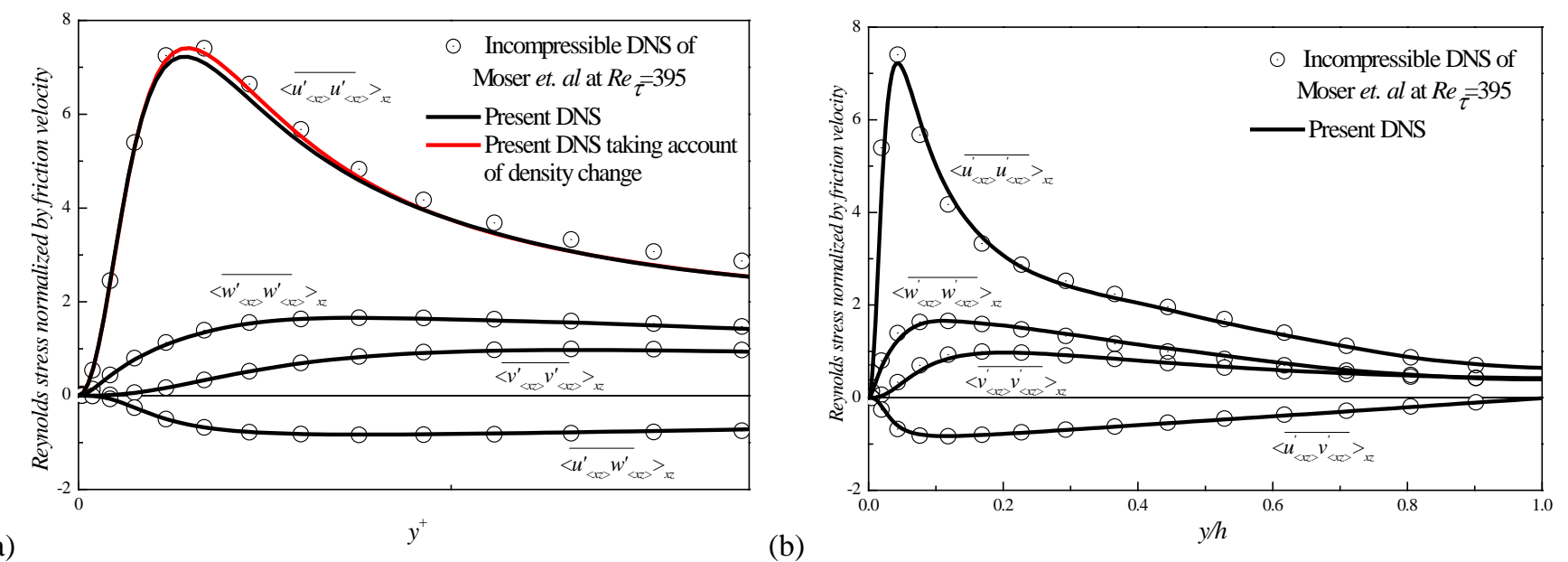

Fig. 3 Comparison of Reynolds stresses in the inner scaling (a) and the outer scaling (b)

\subsection{Coherent Structures}

The turbulent coherent structures developed in the lower half of the channel are visualised using the swirling strength $\left(\lambda_{c i}\right)$ iso-surfaces (see the following references [41-43] for its expression), coloured by the instantaneous streamwise velocity $u$ (see Fig. 4 for all the cases). It is found that the turbulent coherent structures are enhanced above the IPC strips and suppressed above the OPC strips. The turbulent coherent structures close to the lower wall of the channel undergo a redistribution process because of the topography configuration (see in Fig. 1 (a)). Some non-uniformity in the spanwise direction is therefore created. The width of the strips has a strong influence on the distribution of the turbulent coherent structures. Fig. 4 shows that the wider the strips, the better the suppression and enhancement of the turbulent coherent structures. Assuming that similar suppression and enhancement of turbulence are achieved for Cases Nstrip2 and Nstrip4, the level of non-uniformity is higher for Case Nstrip4 because the flow field changes within a narrower space than for Case Nstrip2. A quantitative detailed analysis of all the cases using SADS control is presented in the next section.

(a)

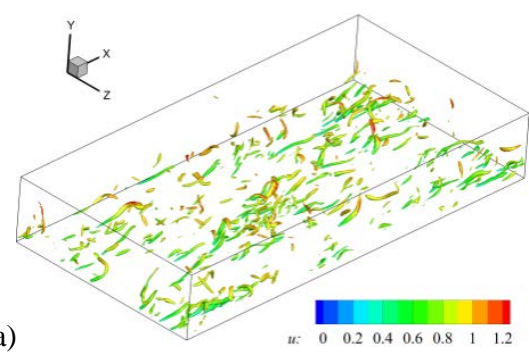

(b)

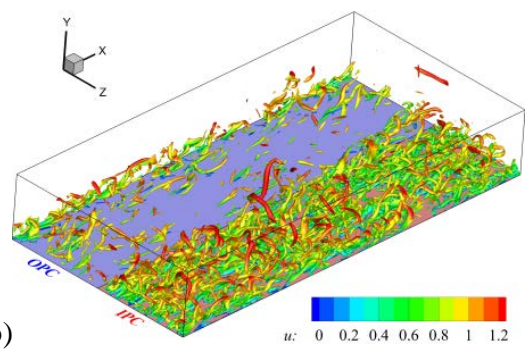

(c)

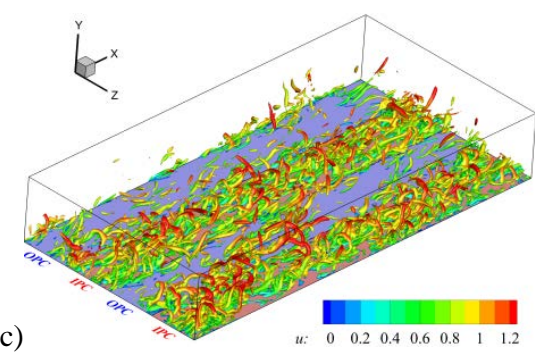



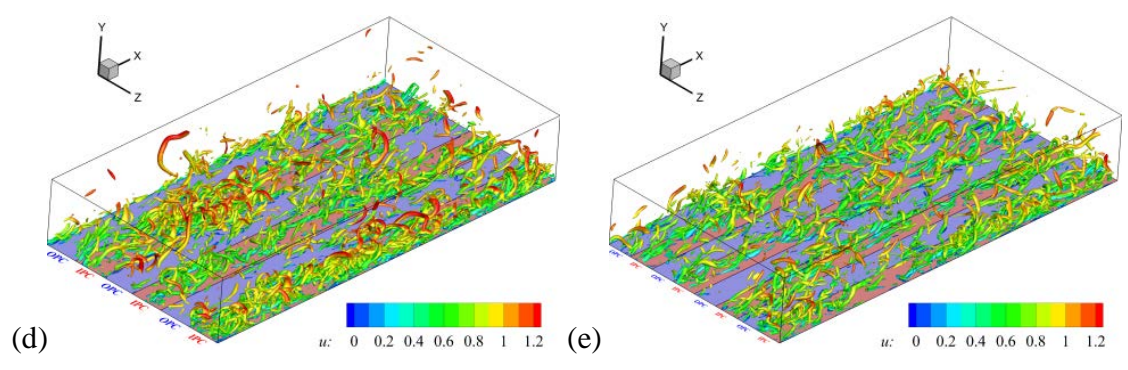

Fig. 4 Turbulent coherent structures visualised using swirling strength iso-surfaces and coloured by the instantaneous streamwise velocity $u$. The strips coloured in blue and red at the lower wall of the channel represent the OPC and IPC regions respectively. (a) Case NC; (b) Case Nstrip2; (c) Case Nstrip4; (d) Case Nstrip6; (e) Case Nstrip8.

\subsection{Mean Flow}

The mean flow field statistics are presented in this section. The skin friction coefficient is first computed. It is based on the streamwise and time averaged statistics and used to show the effect of the strip width on the flow. The mean skin friction coefficient $C_{f}$ is defined as,

$$
C_{f}(z)=\frac{\mu_{w} \partial\langle\bar{u}\rangle_{x} /\left.\partial y\right|_{w}}{0.5 \rho_{b u l k} u_{b u l k}^{2}}
$$

For all the controlled cases, the mean skin friction distribution in the spanwise direction is different from the one observed for the unperturbed case (see Fig. 5 (a)). In general, the skin friction coefficient is slightly reduced over the OPC strips whereas it rises to a large extent over the IPC strips due to the increase in local turbulence intensity. The influence of the strip width on the peak value of $C_{f}$ is clearly observed in Fig. 5 (a). Cases Nstrip6 and Nstrip8, which have narrower strips, exhibit a smaller peak value of $C_{f}$ (about 0.017 and 0.014 respectively) over the IPC strips whilst Cases Nstrip2 and Nstrip4 show larger peak values of $C_{f}$ (about 0.019 and 0.020 respectively). This observation is consistent with the analysis of the coherent structures presented in Fig. 4, in which Cases Nstrip2 and Nstrip4 show a similar trend of turbulent coherent structure enhancement above the IPC strips, while Cases Nstrip6 and Nstrip8 have a weaker effect in impacting turbulence. The result also indicates that this type of control method has a requirement on the width of the strips. The substantial spanwise skin friction reorganisation induces a vertical shear layer between the OPC and IPC strips, resulting in an increase in momentum transport along the spanwise direction. The global change in $C_{f}$ of Cases Nstrip2, 4, 6 and 8 is increased by $68.50 \%, 84.53 \%, 56.77 \%$ and $35.42 \%$ against case NC, respectively. Although $C_{f}$ is greatly enhanced by SADS control, the contribution of the control method to the total drag, including the skin friction and pressure drag, will be checked in the succeeding research of backward-facing ramp flow with a boundary layer separation. In addition, the distribution of the mean skin friction on the upper wall of Case Nstrip4 is presented in Fig. 5 (a), from which we can observe that a higher $C_{f}$ on the upper wall of controlled case is obtained than that of the unperturbed case. This can be explained by the change of the mean streamwise velocity profile across the channel as shown in Fig. 6 (a). The enhanced momentum transport across the channel due to the 
applied SADS has caused a lower mass flux in the lower half of the channel. Therefore, the velocity in the upper half of the channel will be relatively increased due to the mass flow rate being constrained. As a consequence, the peak of mean velocity has been shifted towards the upper wall and the skin friction on the upper wall of Case Nstrip4 is increased as shown in Fig. 6 (a) and Fig. 5 (a) respectively. The present control strategy has indeed influenced the flow field of the upper half of the channel. As a proof-of-concept research in the channel flow, the effect of SADS control on the entire channel will be not discussed in detail in the present paper. The spanwise skin friction gradient $\frac{\mathrm{d} C_{f}(z)}{\mathrm{d} z}$ is calculated to illustrate the effect of SADS control on the strength of the spanwise shear (see Fig. 5 (b)). It can be seen that it reaches its absolute maximum value between the OPC and IPC strips for all the cases and Case Nstrip4 gives the biggest peak among all cases, further indicating its best control effect.

(a)

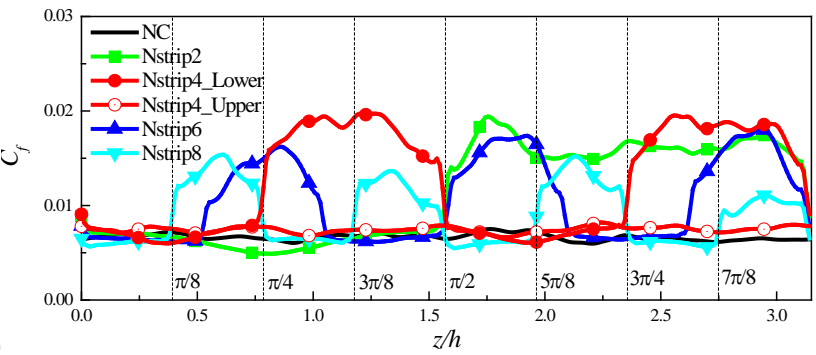

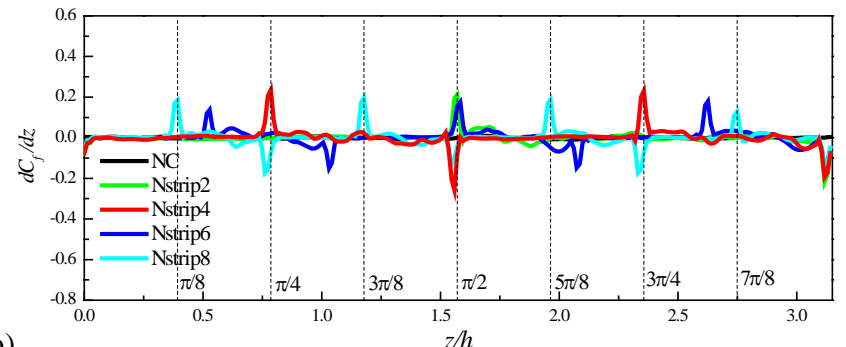

(b)

Fig. 5 Mean skin friction coefficient $C_{f}$ (a) and its spanwise gradient (b) based on $x$ - and time-averaging

The streamwise, spanwise and time averaged velocity profiles $\langle\bar{u}\rangle_{x z}$, normalised by the local bulk velocity and friction velocity respectively are presented in Fig. 6 (a) and (d). The symmetry of the mean streamwise velocity profile observed for Case NC is broken by the control method. The location of the maximum of $\langle\bar{u}\rangle_{x z}$ is shifted from the central line towards the upper wall due to the enhanced turbulent transport at the lower wall. It also varies non-monotonically with the width of the strips in the near-wall region as illustrated in Fig. 6 (b). With the decrease of the width of the strip, the mean velocity in the near-wall region firstly increases and then decreases, giving a maximum increase of the near-wall velocity for Case Nstrip4. Consequently, the velocity gradient in Case Nstrip4 gets its maximum value close to the controlled wall of the channel and yields the largest skin friction as observed in Fig. 5. This phenomenon is also exhibited when showing the mean streamwise velocity profiles normalised by the local friction velocity, i.e. $\left\langle\overline{u_{\tau}}\right\rangle_{x z}$ (see Fig. 6 (d)). All the velocity profiles from the cases with SADS control are shifted downward as compared to those of Case NC. This is a typical characteristic of the velocity profile in drag increasing turbulent flows [36]. As expected, Case Nstrip4 exhibits the largest downward shift among all the studied cases. 

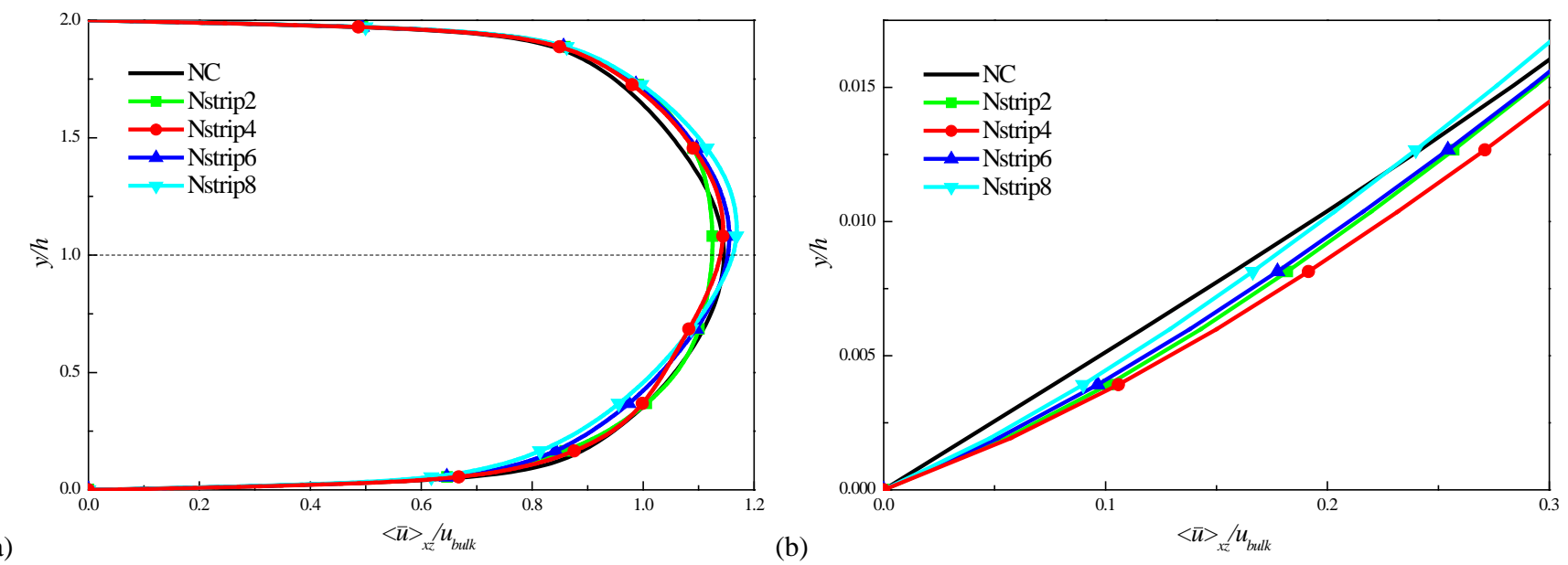

(a)

(b)
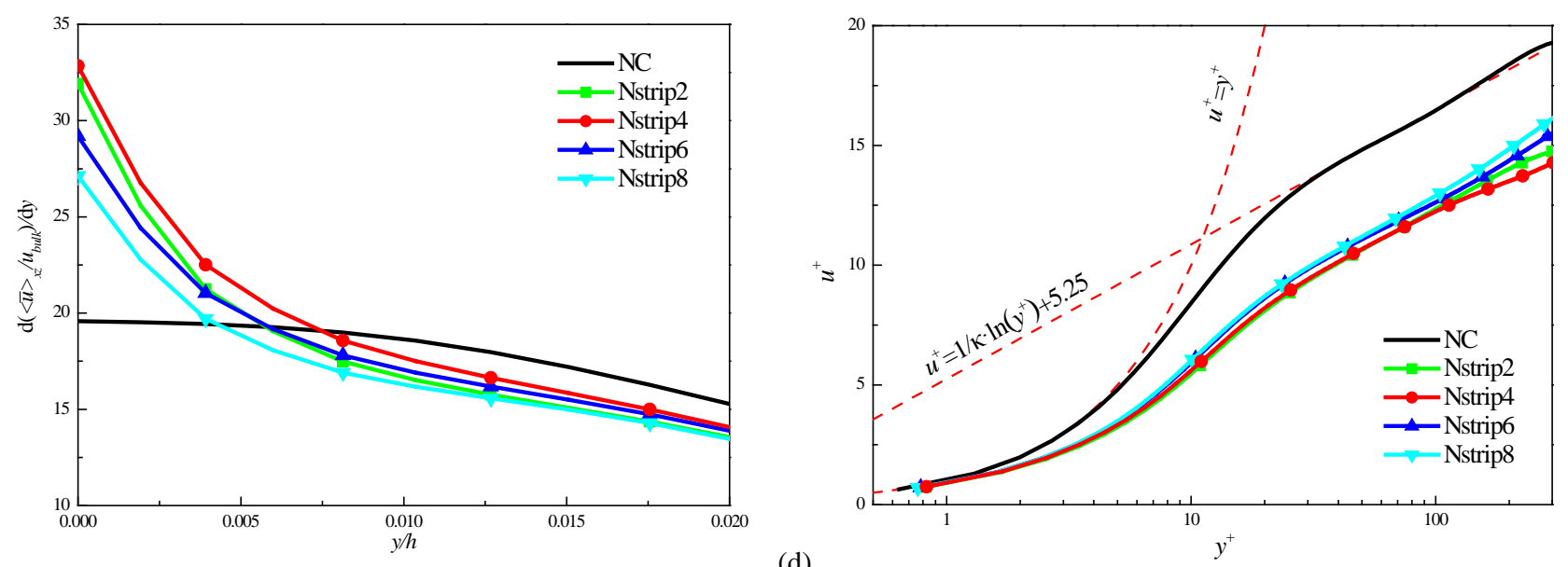

(c)

(d)

Fig. 6 Mean streamwise velocity profile $\langle\overline{\boldsymbol{u}}\rangle_{x \boldsymbol{z}}$ in the outer scaling normalised by the bulk velocity (a) (zoomed in (b) for the near-wall region) and its wall-normal gradient (c) as well as in the inner scaling normalised by the local friction velocity (d). They are presented for each case.

In addition, contours of the mean streamwise velocity component $\langle\bar{u}\rangle_{x}$ with the mean velocity vector $\left(\langle\bar{w}\rangle_{x},\langle\bar{v}\rangle_{x}\right)$ plotted in the $y$-z plane are compared in Fig. 7 (left-hand side) to show the influence of the SADS control on the distribution of the mean streamwise velocity component in the spanwise direction. SADS modulation of the flow field is very clear in Fig. 7. Low speed regions are induced above the OPC strips, which resemble large-scale low speed streaks. In contrast, the near-wall velocity increases above the IPC strips. The simulated spanwise distribution of the local mean velocity is qualitatively in good agreement with the experimental observations of Nugroho et al. [9] and Mejia-Alvarez et al. [11]. The former imposed regularly distributed converging and diverging riblet-type surface roughness to a zero-pressure-gradient turbulent boundary layer flow and the latter identified the same phenomenon and called it spanwise-localised LMP bounded by HMP. These mean flow heterogeneities are locked due to the periodic nature of the imposed OPC/IPC strips in Cases Nstrip2 and Nstip4. While the width of the strips is further reduced in Cases Nstrip6 and Nstrip8, the spanwise modulation of the mean streamwise velocity profiles gets weaker, as indicated in Fig. 7 (g) and (i). 
To illustrate the redistribution of the mean velocity field by SADS control, the mean streamwise velocity fluctuation relative to the spanwise averaged value $\langle\bar{u}\rangle_{x}-\langle\bar{u}\rangle_{x z}$ in the $y-z$ plane is presented in Fig. 7 (right-hand side). According to the definition previously given in Subsection 3.1, $\langle\bar{u}\rangle_{x}-\langle\bar{u}\rangle_{x z}$ can be rewritten as $\langle\bar{u}\rangle_{x_{\langle z\rangle}}$, highlighting the spanwise fluctuations due to SADS control. Fig. 7 (f) shows that the fluid above the IPC and OPC strips is accelerated and decelerated respectively up to more than $10 \%$ of the local mean velocity $\langle\bar{u}\rangle_{x z}$; the alternatively distributed high- and low-speed regions extend to the half a height of the channel, indicating the existence of large-scale flow structures. As illustrated in Fig. 7 (h) and (j), the spanwise variation fades away when the width of each strip gets smaller. In the present study, the LSSVs, which are generated by the control strategy, are closely linked to the spanwise variation induced by SADS control, leading to the enhancement of the momentum transport. These large-scale flow structures, namely the high/low steady velocity streaks generated by Cases Nstrip2 and Nstrip4 are different from the near-wall velocity streaks observed in the instantaneous flow field. Mejia-Alvarez et al. [11] suggested that these large-scale high/low mean velocity streaks could be the preferred pathways for developing instantaneous large-scale motions, namely HMRs and LMRs.

(a)

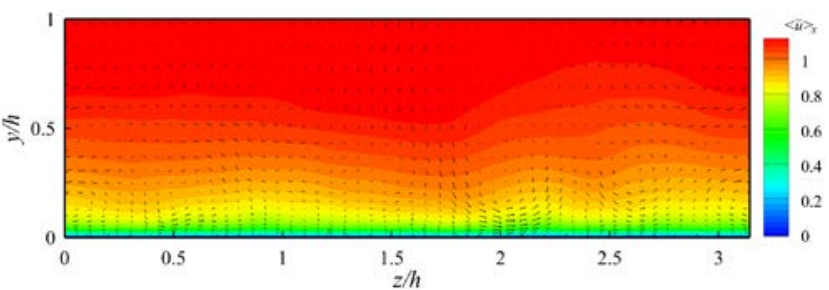

(c)

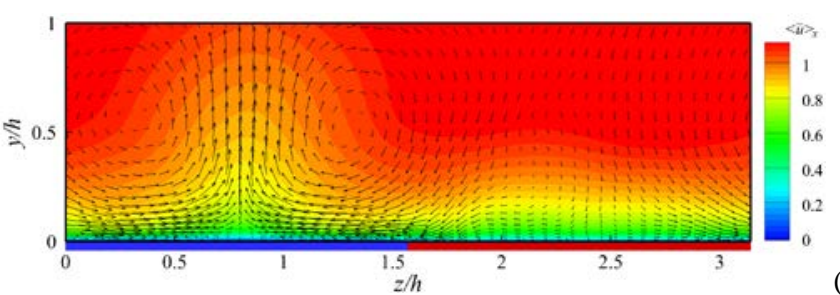

(d)

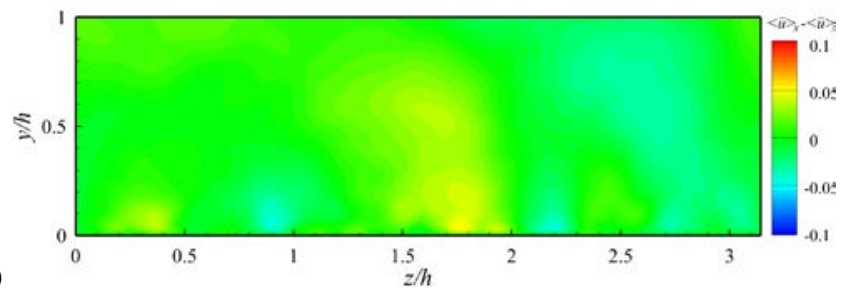

(b)

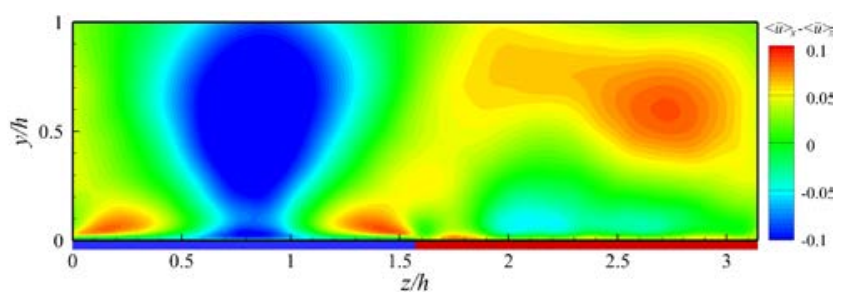

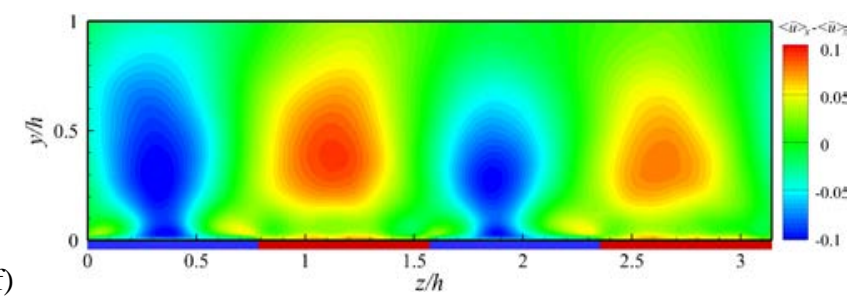

(f)

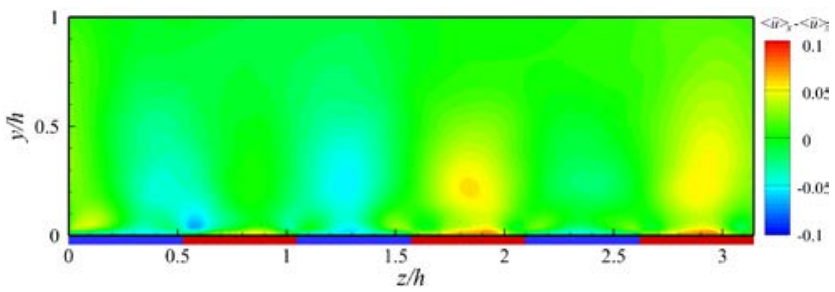

(h) (g)

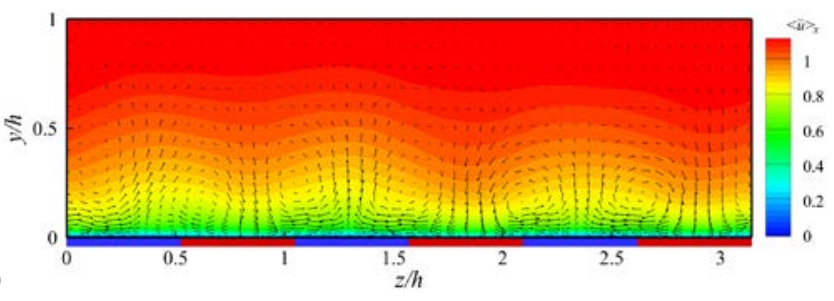


(i)

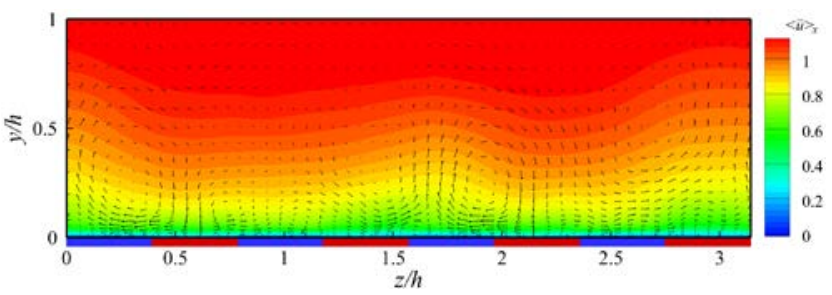

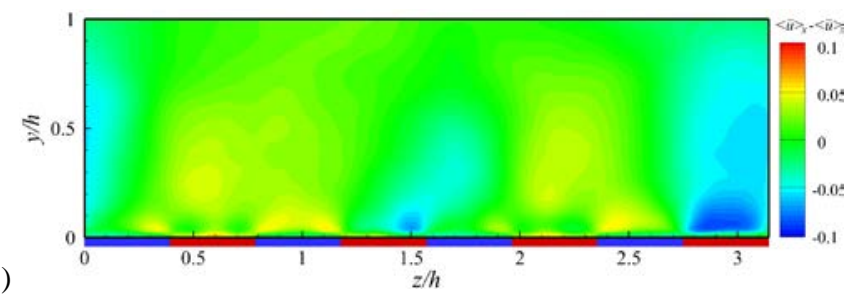

Fig. 7 Distribution of the $x$ - and time-averaged streamwise velocity component $\langle\overline{\boldsymbol{u}}\rangle_{x}$ with the vector $\left(\langle\overline{\boldsymbol{w}}\rangle_{x},\langle\overline{\boldsymbol{v}}\rangle_{x}\right)$ (left-hand side of the figure) and mean velocity fluctuations relative to its spanwise averaged value $\langle\overline{\boldsymbol{u}}\rangle_{x}-\langle\overline{\boldsymbol{u}}\rangle_{x \boldsymbol{z}}$ (right-hand side of the figure) from (a) to (j). The results come from Case NC, Nstrip2, Nstrip4, Nstrip6 and Nstrip8 from top to bottom. For each plot, the blue and red strips plotted along the z-coordinate axis represent the regions controlled by OPC and IPC respectively. The distribution of the mean streamwise velocity from Cases Nstrip2 and Nstrip4 are in agreement with the results of the rough wall experiment E1 from Nugroho et al. [9] (Page 94, Fig. 3 (a)) and the flow over Case B6 from Mejia-Alvarez et al. [11] (Page 025111-7, Fig. 2 (c)).

Given that the largest spanwise variation is generated in Case Nstrip4 and following the skin friction coefficient analysis (see Fig. 5) and the distribution of the mean streamwise velocity in $y$-z plane (see Fig. 7), this case will be focused on until the end of this subsection. The mean streamwise velocity profiles $\langle\bar{u}\rangle_{x}$ located at four representative positions within one pair of OPC and IPC strips are plotted in Fig. 8. The spanwise averaged velocity profiles, namely $\langle\bar{u}\rangle_{x z}$, are presented and Case NC is used as a reference. The velocity profiles in blue and red in the figure are extracted from the middle positions of the OPC and IPC strips respectively, whereas the two other positions are located at the interfaces between the OPC and IPC strips. It can be seen from Fig. 8 (a) that the blue velocity profile is shifted upwards because the turbulence motion is suppressed by the OPC, whereas the fluid located above the middle of the IPC strip is accelerated over the whole half height of the channel. The velocity profiles situated at the interfaces between the OPC and IPC strips are less modified than the other two and exhibit a similar distribution as the spanwise averaged value $\langle\bar{u}\rangle_{x Z}$ of Case Nstrip4. It can be seen from Fig. 8 (a) that there exists a discrepancy of the velocity profiles at between the IPC/OPC interface and OPC/IPC interface. We guess that the large-scale structures in the channel whose size might be greater than the size of the strip, resulting in the statistics being not symmetric. The similar phenomenon can also be seen in the study of Hansen et al. [37], who aim to suppress flow separation of the airfoil using wavy leading edge. They suggest that the symmetry of statistics is lost when large-scale vortex is generated. In the present study, with the reduction of the size of the strip, the discrepancy of the profiles will also be greater, due to the scale of LSSV becomes relatively larger than the distance between interfaces. With a close check of the time averaged flow field, we can confirm that the flow is not symmetric against the interface locations. According to the time averaged velocity field in Fig. 7 (e), the peak of the low-speed region above the OPC strip is also not at the centre of the two interfaces. As shown in Fig. 11 (c), the IPC/OPC interface is much closer to the centre of the LSSV but the OPC/IPC interface is relatively far from the centre of the LSSV. The velocity above the IPC strip is higher than that at other locations due to the stronger turbulence generated above the IPC strip. This is in agreement with the distribution of $C_{f}$ (see Fig. 5), in which the maximum $C_{f}$ is reached around the middle of the IPC. This is also confirmed when plotting the velocity profiles in 
the inner scaling as illustrated in Fig. 8 (b). A clear downward shift of the log-layer is seen in Fig. 8 (b) for every profile except the one in the middle of the OPC strip. As indicated in Fig. 8 (b), the velocity profile extracted from the middle of the IPC region shows the maximum downward shift, whilst the one located in the middle of the OPC strip exhibits the minimum downward shift. The downward shifts of the two profiles located at the interfaces are situated between the blue and red lines. The log-law layer of the velocity profile in the middle of the OPC region (in blue) is very close to that of the unperturbed Case NC.
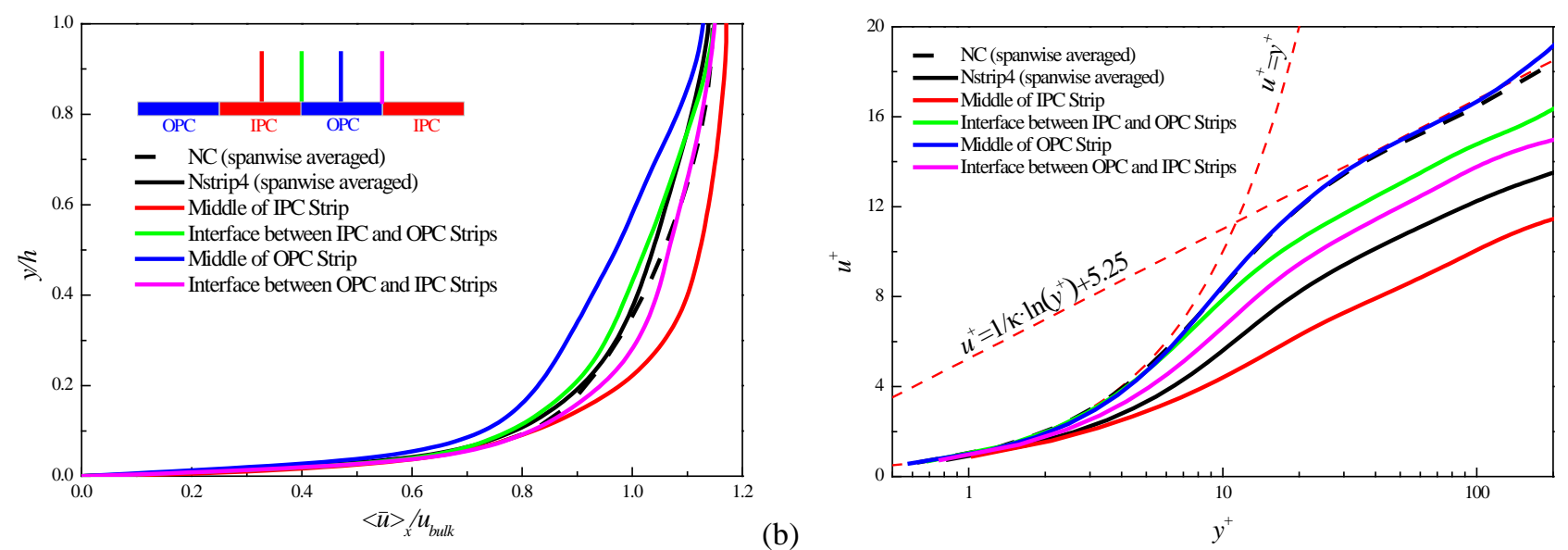

(a)

(b)

Fig. 8 Mean streamwise velocity profiles extracted from four representative positions within one pair of OPC and IPC strips. They are shown in the outer (a) and the inner (b) scaling.

Iso-contours of the mean swirling strength $\Lambda_{c i}=\left(\omega_{x} /\left|\omega_{x}\right|\right)\left\langle\overline{\lambda_{c l}}\right\rangle_{\langle x\rangle}$ of Case Nstrip4 are presented in Fig. 9to identify the large-scale motions generated by the SADS control strategy. For Case Nstrip4, a clear distribution of $\Lambda_{c i}$ is identified. Largescale negative (resp. positive) $\Lambda_{c i}$ clouds are located at the left (resp. right) hand sides of the OPC strips. This indicates that the large-scale steady counter-rotating streamwise vortices are induced and sustained by the control method. They enhance momentum transport throughout the channel. Low speed fluid is transported from the near-wall region to the central part of the channel as shown in Fig. 7 (e). Similar large-scale motions were reported by Barros et al. [40] in a flow with irregular transverse roughness and Willingham et al. [10] in a flow with highly ordered transverse roughness. Recently, Kevin et al. [41] suggest that counter-rotating vortices observed in the time-average view is not representative of the instantaneous vortex arrangement induced by the surface texture. They point out that the large vortical events that give rise to the time-average secondary flow are to some extent intermittent in time. Therefore, from the statistical perspective, it can be concluded from Fig. 9 that the large-scale statistically steady counter-rotating streamwise vortices, whose cores are located between the OPC and IPC strips, are generated by the SADS control. Further, it can be seen from Fig. 9 that the IPC/OPC interface is much closer to the centre of the LSSV whereas the OPC/IPC interface is relatively far from the centre of the LSSV. The similar phenomenon can also be seen in the study of Hansen et al. [42], who aim to suppress flow separation of airfoil using wavy 
leading edge. They suggest that the symmetry of the statistics will be lost when large-scale vortex is generated. According to the time-average streamwise velocity field as illustrated in Fig. 7(e), the peak position of the low-speed region above the OPC strip is not at the centre of the two interfaces as expected.

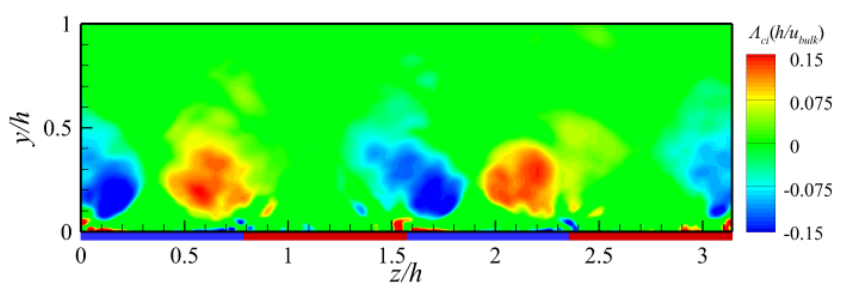

Fig. 9 Distribution of the swirling strength $\boldsymbol{\Lambda}_{\boldsymbol{c} \boldsymbol{i}}$ normalized by $\left(\boldsymbol{u}_{\boldsymbol{b u l k}} / \boldsymbol{h}\right)$ for Case Nstrip4. The swirling strength depends on the sign of the streamwise vortex $\omega_{x}$.

\subsection{Turbulent Kinetic Energy and Reynolds Stress}

The turbulence kinetic energy and Reynolds shear stress normalised by the local friction velocity are plotted for the five cases, as a function of $y / h$ in Fig. 10. They are calculated from the time-, $x$ - and $z$-averaged statistics as,

$$
T K E=\frac{1}{2}\left\langle\overline{\left(u_{\imath}-\left\langle\bar{u}_{\imath}\right\rangle_{x z}\right)\left(u_{\imath}-\left\langle\overline{u_{\imath}}\right\rangle_{x z}\right)}\right\rangle_{x z} /\left\langle\overline{u_{\tau}}\right\rangle_{x z}^{2}
$$

and

$$
R S S=\left\langle\overline{\left(u-\langle\bar{u}\rangle_{x z}\right)\left(v-\langle\bar{v}\rangle_{x z}\right)}\right\rangle_{x z} /\left\langle\overline{u_{\tau}}\right\rangle_{x z}^{2}
$$

Fig. 10 (a) shows that, for the unperturbed case, TKE reaches a peak value around $y / h \approx 0.05$. Compared with Case NC, the peak values of TKE of all the controlled cases vary non-monotonically with the width of the strips. The smallest peak value is achieved for Case Nstrip4 due to the maximum skin friction as indicated in Fig. 5 (a). Further examining the TKE profile in the near-wall region, it shows that the peak value positions of all the controlled cases move towards the wall with the Case Nstrip4 being the closest to it. This indicates that the strip distribution used in Case Nstrip4 corresponds to the most optimised configuration for a plane channel flow at $R e=6,000$. The RSS term plays a key role in the turbulence momentum transport and turbulence energy production, and its minimum value (see Fig. 10 (c)) is obtained at the same location as TKE maximum value (see Fig. 10 (a)). The RSS profiles in the near-wall region are further examined in a zoomed view (see Fig. 10 (d)). A rapid decrease of RSS occurs in the region defined by $0<y<0.02 h$ for the cases with SADS control, which indicates that the near-wall momentum transport is largely enhanced. This result is consistent with the TKE increase observed in the same region. 


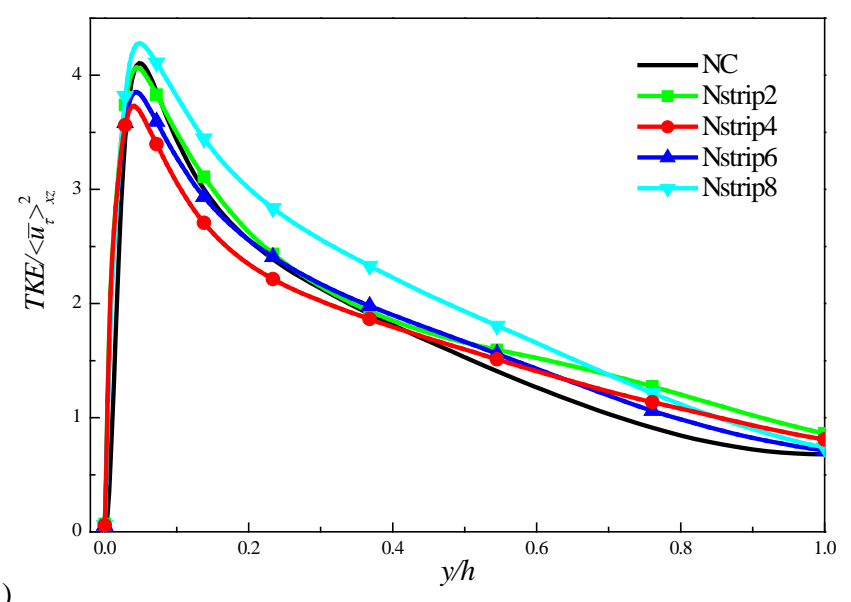

(a)

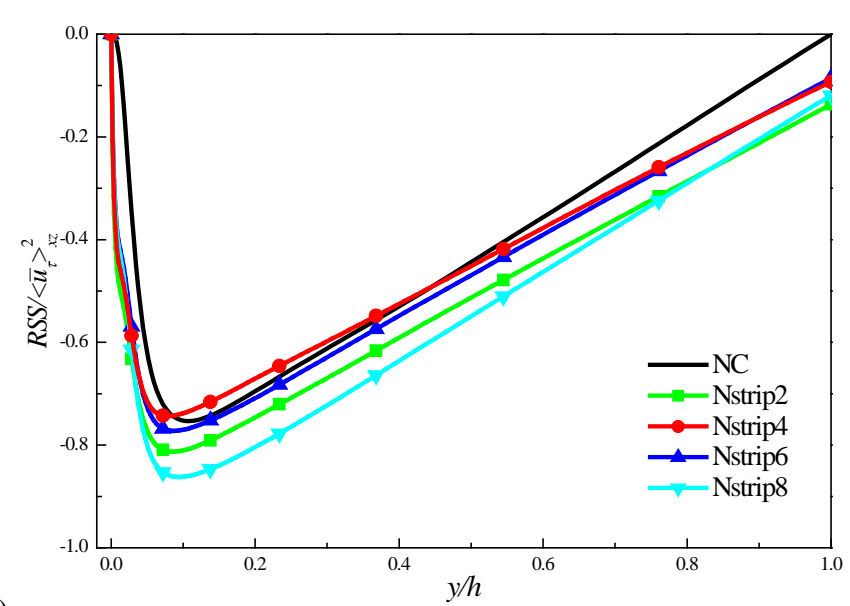

(c)

(d)

(b)
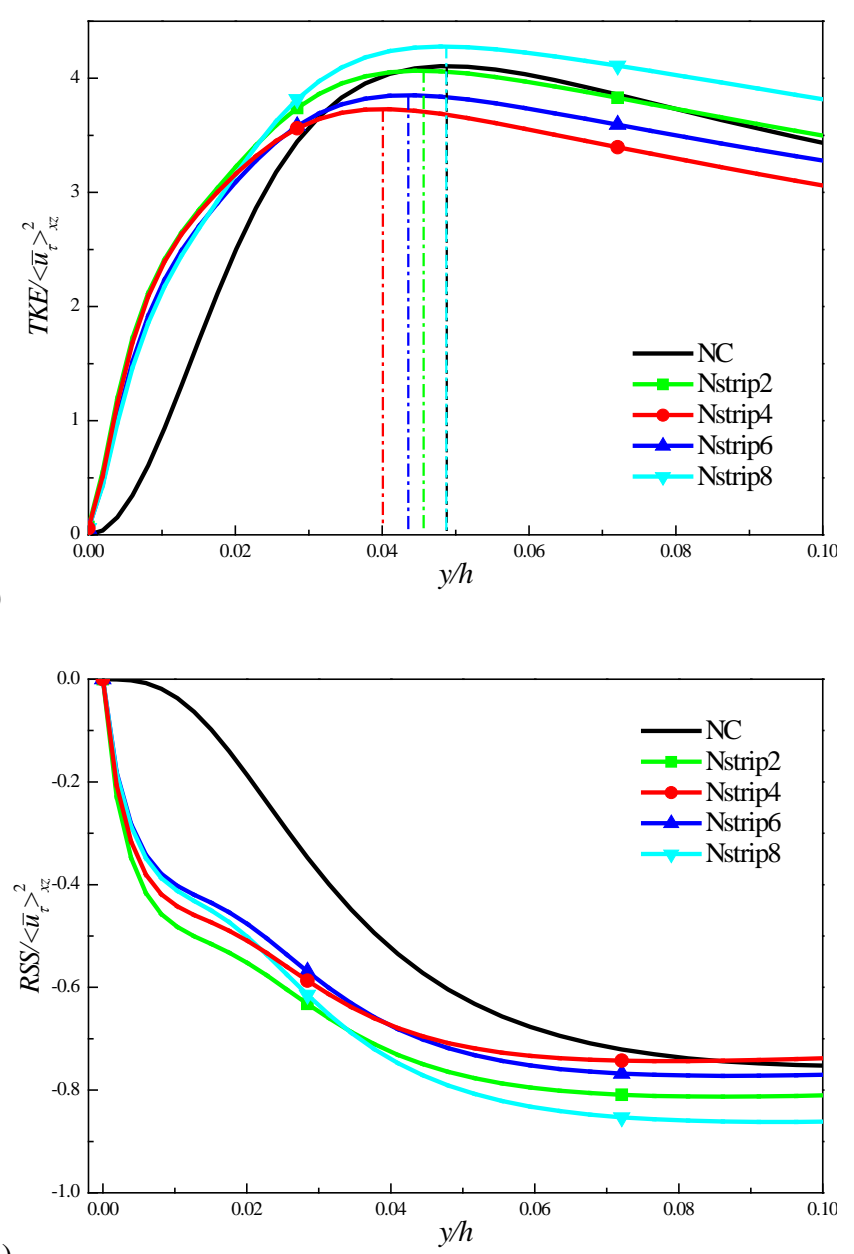

Fig. 10 Turbulence kinetic energy (a) and Reynolds shear stress (c) calculated by time-, $x$ - and $z$-averaged statistics for all the cases. A zoom of the near-wall behaviour of TKE and RSS is presented in (b) and (d) respectively.

The TKE and RSS distributions based on the time- and $x$-averaged statistics (denoted TKE $\left.\right|_{x t}$ and RSS $\left.\right|_{x t}$ respectively) as well as the mean velocity vector $\left(\langle\bar{w}\rangle_{x},\langle\bar{v}\rangle_{x}\right)$ in the $y$-z plane for Case Nstrip4 are presented in Fig. 11 in order to study the properties of LSSVs in conjunction with momentum transport. TKE $\left.\right|_{x t}$ and $\left.R S S\right|_{x t}$ are expressed as $\frac{1}{2}\left\langle\overline{u_{l,\langle x\rangle}^{\prime}} u_{l,\langle x\rangle}^{\prime}\right\rangle_{x}$ and $\left\langle\overline{\left\langle u_{\langle x\rangle}^{\prime} v_{\langle x\rangle}^{\prime}\right.}\right\rangle_{x}$ respectively. They are normalised by the square of the bulk velocity $u_{b u l k}^{2}$. “ $\left.\right|_{x t}$ ” stands for the fluctuation calculated by subtracting the $x$ - and time-averaged velocity from the instantaneous quantity. TKE $\left.\right|_{x t}$ and $\left.R S S\right|_{x t}$ clearly exhibit large-scale patterns in Case Nstrip4. Resembling that the streamwise vortices near the wall act a dominant role in turbulence production and momentum transfer [37,38], the large-scale motions generated by SADS control modulate the distribution of TKE an RSS. Both TKE $\left.\right|_{x t}$ and $\left.R S S\right|_{x t}$ are influenced by large-scale ejection and sweep motions. The ejection motion (marked by the two white arrows in Fig. 11 (a) and (b)) transports the low momentum fluid from the near-wall region into the outer part of the channel and therefore produces higher $\left.T K E\right|_{x t}$ and $\left.R S S\right|_{x t}$ in the outer region. Conversely, the sweep motion brings high momentum fluid from the outer part of the channel into the inner layer as shown in Fig. 7 (e), leading to higher $\left.T K E\right|_{x t}$ 
and $\left.R S S\right|_{x t}$ in the near-wall region. Because the LSSVs core is roughly centered at the interfaces between the OPC and IPC strips, the ejection and sweep motions mainly happen above the OPC and IPC strips respectively. These are consistent with the high $\left.T K E\right|_{x t}$ and $\left.R S S\right|_{x t}$ regions in the outer part and the near-wall part of the channel. Similar observations are also obtained by Nugroho et al. [9], Barros et al. [40] and Willingham et al. [10]. It is worth noting that the sweep motions confine high $\left.T K E\right|_{x t}$ close to the near-wall region above the IPC strips, resulting in the reduction of $\left.T K E\right|_{x t}$ over the major part of the channel. This phenomenon is also reported by Nugroho et al. [9]. $\left.R S S\right|_{x t}$ behaves in a similar way as TKE $\left.\right|_{x t}$ (see Fig. 11) and the spanwise distribution of alternated low/high $\left.T K E\right|_{x t}$ and $\left.R S S\right|_{x t}$ streaks corresponds to low/high mean streamwise velocity streaks (see Fig. 7 (e)). This means that the ejection and sweep motions are the two major events related to momentum transport. In the wall-normal direction, the penetration depth of the large-scale motions even extends beyond the half height of the channel.

(a)

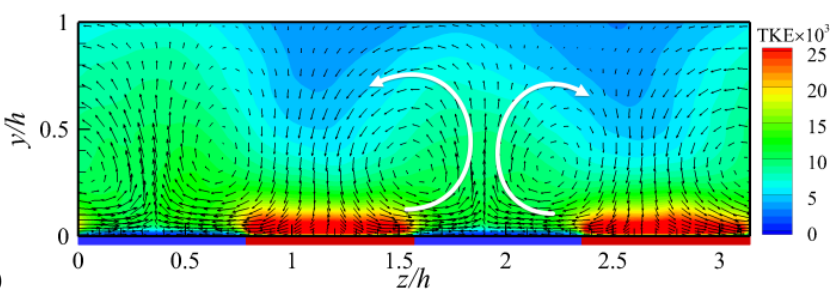

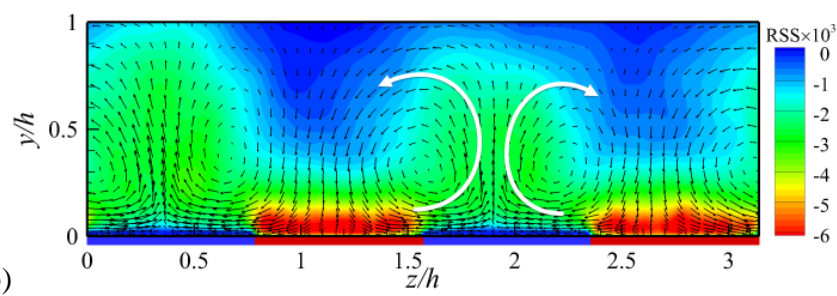

Fig. 11 Distribution of TKE (a) and RSS (b) based on the time- and $x$-averaged statistics for Case Nstrip4. The vector $\left(\langle\overline{\boldsymbol{w}}\rangle_{x},\langle\overline{\boldsymbol{v}}\rangle_{x}\right)$ is plotted in the $y$-z plane. The white arrows represent the local sweep and ejection motions. The distribution of TKE from Case Nstrip4 corresponds to the results of the rough wall experiment E1 from Nugroho et al. [9] (Page 94, Fig. 3 (a)) and the flow over Case B6 from Mejia-Alvarez et al. [11] (Page 025111-7, Fig. 2 (c)).

To study Case Nstrip4 in more details, the following Reynolds stresses $\left\langle\overline{u_{l,\langle x\rangle}^{\prime 2}}\right\rangle_{x}(i=1,2,3)$ and $\left\langle\overline{\left\langle u_{\langle x\rangle}^{\prime} v_{\langle x\rangle}^{\prime}\right.}\right\rangle_{x}$ are extracted from four representative positions (see Fig. 8 for their definition) within one pair of the OPC and IPC strips, normalised by the local friction velocity. Fig. 12 (a) shows that the intensity of the streamwise component of the Reynolds normal stress $\left\langle\overline{\left\langle u_{\langle x\rangle}^{\prime} u_{\langle x\rangle}^{\prime}\right.}\right\rangle_{x}$ decreases in the near-wall region above the middle of the OPC strip whereas it increases intensively over the IPC region. The near-wall distributions of the wall-normal and spanwise components are depicted in Fig. 12 (b) and (c) respectively. The wall-normal component of the Reynolds stresses on the lower wall of the channel is not equal to zero because of the imposed control strategy. The RSS and the turbulence intensity exhibit a similar pattern in the near-wall region as indicated in Fig. 12. All the components of the Reynolds stresses in the outer part of the channel above the OPC strip are largely enhanced by the ejection motion induced by LSSVs. The sweep motion confines high turbulence intensity and RSS in the near-wall region above the IPC strip, which is in an agreement with the distribution of TKE and RSS in the $y$-z plane as indicated in Fig. 11 (c) and (d). 

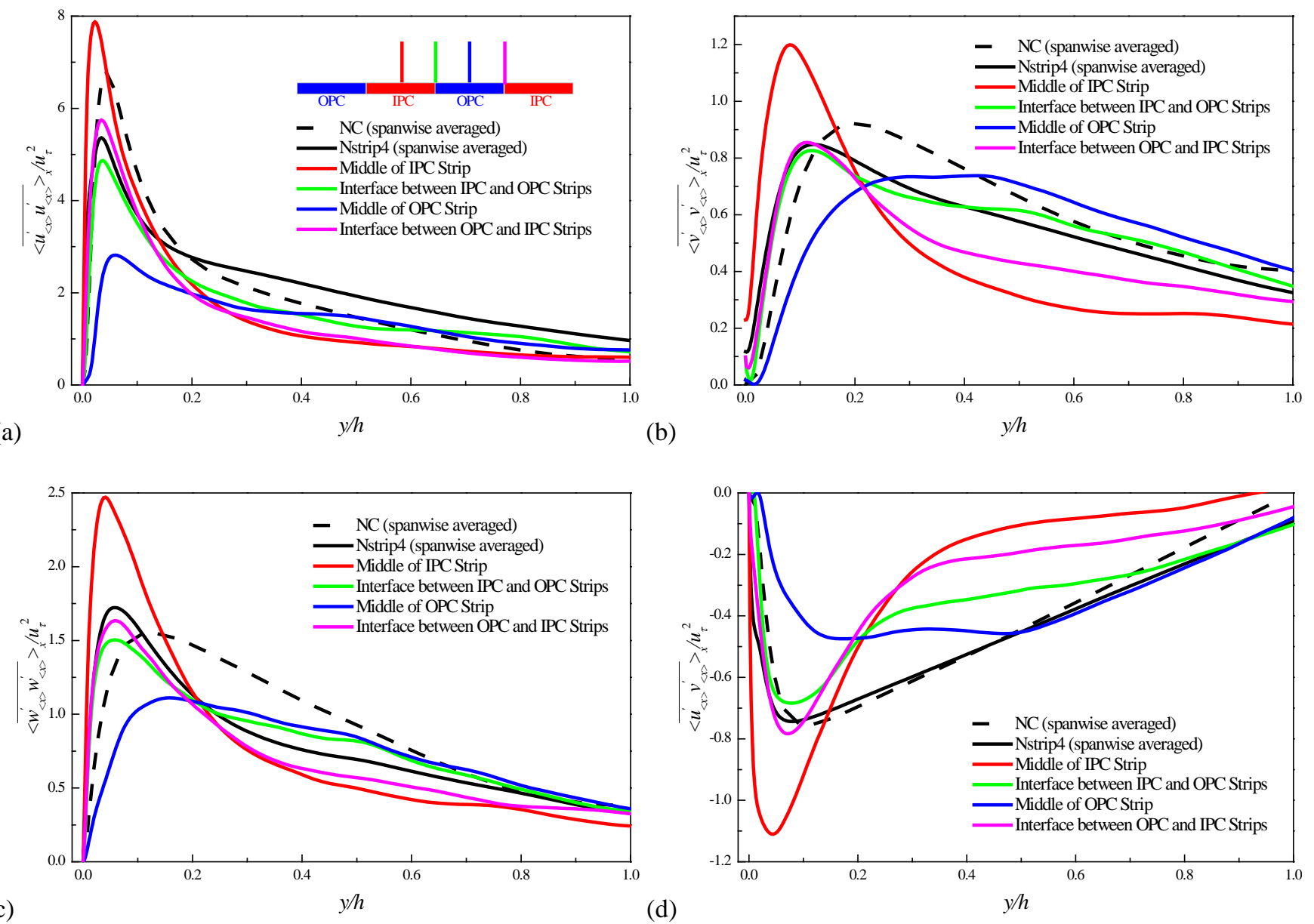

Fig. 12 Reynolds stresses $\left\langle\overline{\left\langle\boldsymbol{u}_{\boldsymbol{\nu},\langle x\rangle}^{\prime} \boldsymbol{u}_{\boldsymbol{\imath},\langle x\rangle}^{\prime}\right.}\right\rangle_{\boldsymbol{x}}$ and $\left\langle\overline{\left\langle\boldsymbol{u}_{\langle x\rangle}^{\prime} \boldsymbol{v}_{\langle x\rangle}^{\prime}\right.}\right\rangle_{\boldsymbol{x}}$ extracted from four representative positions within one pair of OPC and IPC strips,

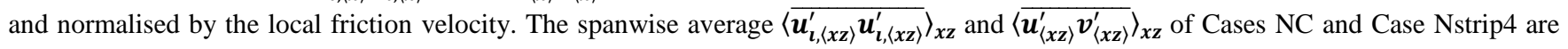
plotted for comparison.

Two other quantities, namely $\left\langle\left(u_{i,\langle x z\rangle}^{\prime}-u_{i,\langle x\rangle}^{\prime}\right)^{2}\right\rangle_{z}$ and $\left\langle\left(u_{\langle x z\rangle}^{\prime}-u_{\langle x\rangle}^{\prime}\right)\left(v_{\langle x z\rangle}^{\prime}-v_{\langle x\rangle}^{\prime}\right)\right\rangle_{z}$ are defined to emphasise the influence of the distribution of the strips in Case Nstrip4 on the flow field, in the spanwise direction. They read:

$$
\left\langle\left(u_{i,\langle x z\rangle}^{\prime}-u_{i,\langle x\rangle}^{\prime}\right)^{2}\right\rangle_{z}=\left\langle\left(\left\langle\bar{u}_{\imath}\right\rangle_{x}-\left\langle\overline{u_{l}}\right\rangle_{x z}\right)^{2}\right\rangle_{z}
$$

and

$$
\left\langle\left(u_{\langle x z\rangle}^{\prime}-u_{\langle x\rangle}^{\prime}\right)\left(v_{\langle x z\rangle}^{\prime}-v_{\langle x\rangle}^{\prime}\right)\right\rangle_{z}=\left\langle\left(\langle\bar{u}\rangle_{x}-\langle\bar{u}\rangle_{x z}\right)\left(\langle\bar{v}\rangle_{x}-\langle\bar{v}\rangle_{x z}\right)\right\rangle_{z},
$$

where the fluctuations are calculated by subtracting the $x$-, $z$ - and time-averaged velocity from the $x$-and time-averaged one. They are normalised by $\left\langle\overline{\left\langle u_{l,\langle x z}^{\prime 2}\right.}\right\rangle_{x z}$ and $\left\langle\overline{\left\langle u_{\langle x z}^{\prime} v_{\langle x z}^{\prime}\right\rangle}\right\rangle_{x z}$ respectively and computed for $y>0$ in the wall-normal direction. Compared with Case NC, a clear peak value of Reynolds shear stress is exhibited by Case Nstrip4. It is located at about $0.39 \mathrm{~h}$ from the lower wall of the channel. As the new definition of the Reynolds shear stress (see Eqs. 19) shows the contribution of spanwise motions to the momentum transport by subtracting $\langle\bar{u}\rangle_{x z}$ from $\langle\bar{u}\rangle_{x}$, it is reasonable to assume that its peak is induced by the spanwise large inhomogeneous variations created by the alternatively distributed strips control in the spanwise 
direction. Furthermore, as the peak position is found away from the lower wall of the channel, this demonstrates the existence of large-scale motions whose core is located in the central region of the half-height channel. These large-scale motions resemble LSSVs. The momentum transport is enhanced throughout the channel by LSSVs, leading to a significant rise of the streamwise Reynolds normal stress $\left\langle\left(u_{\langle x z\rangle}^{\prime}-u_{\langle x\rangle}^{\prime}\right)^{2}\right\rangle_{z}$ whose peak position is consistent with the Reynolds shear stress. Two other Reynolds normal stresses, i.e. $\left\langle\left(v_{\langle x z\rangle}^{\prime}-v_{\langle x\rangle}^{\prime}\right)^{2}\right\rangle_{z}$ and $\left\langle\left(w_{\langle x z\rangle}^{\prime}-w_{\langle x\rangle}^{\prime}\right)^{2}\right\rangle_{z}$ also increase in comparison with those of Case NC (see Fig. 13). It can be concluded that large-scale counter-rotating streamwise vortices are generated in Case Nstrip4 and that they play a significant role in Reynolds stress production and distribution, as well as in momentum transport.

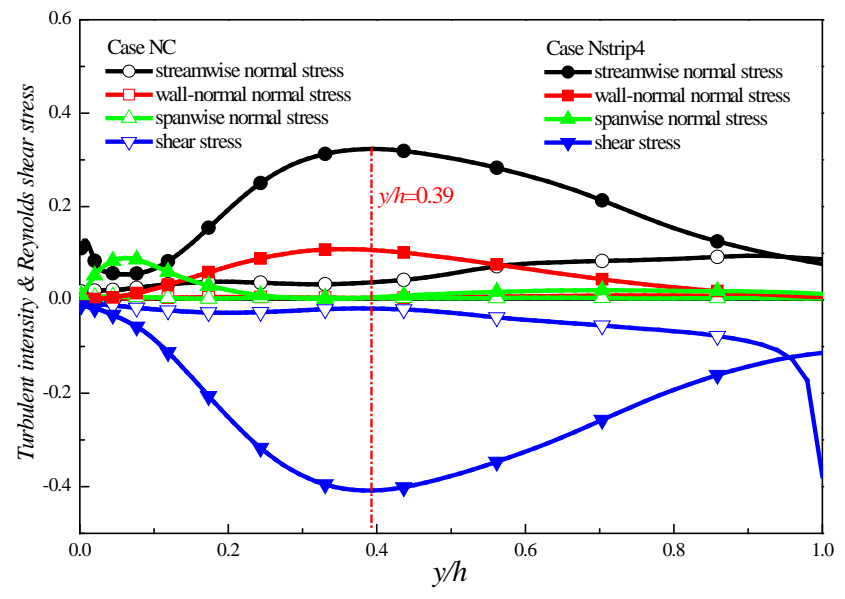

Fig. 13 Reynolds normal stress $\left\langle\left(\boldsymbol{u}_{\boldsymbol{i},\langle x \boldsymbol{z}\rangle}^{\prime}-\boldsymbol{u}_{\boldsymbol{i},\langle\boldsymbol{x}\rangle}^{\prime}\right)^{\mathbf{2}}\right\rangle_{\boldsymbol{z}}$ and Reynolds shear stress $\left\langle\left(\boldsymbol{u}_{\langle x \boldsymbol{z}\rangle}^{\prime}-\boldsymbol{u}_{\langle\boldsymbol{x}\rangle}^{\prime}\right)\left(\boldsymbol{v}_{\langle x \boldsymbol{z}\rangle}^{\prime}-\boldsymbol{v}_{\langle x\rangle}^{\prime}\right)\right\rangle_{\boldsymbol{z}}$ for Cases NC (line with unfilled symbols) and Nstrip4 (line with filled symbols). The definition of $\left\langle\left(\boldsymbol{u}_{\boldsymbol{i},\langle x z\rangle}^{\prime}-\boldsymbol{u}_{\boldsymbol{i},\langle x\rangle}^{\prime}\right)^{\mathbf{2}}\right\rangle_{\boldsymbol{z}}$ and $\left\langle\left(\boldsymbol{u}_{\langle x \boldsymbol{z}\rangle}^{\prime}-\boldsymbol{u}_{\langle x\rangle}^{\prime}\right)\left(\boldsymbol{v}_{\langle x \boldsymbol{z}\rangle}^{\prime}-\boldsymbol{v}_{\langle x\rangle}^{\prime}\right)\right\rangle_{\boldsymbol{z}}$ refers to Equations (18) and (19).

\subsection{Instantaneous Flow Field}

The instantaneous streamwise vorticity $\omega_{x}$ and streamwise velocity $u$ normalised by the friction velocity $u_{\tau}$ are plotted for Cases NC and Nstrip4 in the $y$-z plane in Fig. 14, where LSSVs and their transport effect can be observed. All the slices are extracted from location $x / h=4.8$. According to Fig. 14 (b), the LSSV is identified as a packet of small-scale flow motions with high values of vorticity (see within the dashed line boxes in Fig. 14 (b)), similar to the hairpin packets model of wall turbulence proposed by Adrian et al. [43]. The instantaneous ejection of low speed fluid above the OPC strips (see within the dashed line boxes in Fig. 14 (d)) and the sweep motions of high speed fluid above the IPC strips can also be seen in Fig. 14 (d), which is consistent with the analysis based on the statistics as indicated in Fig. 7 (e). 


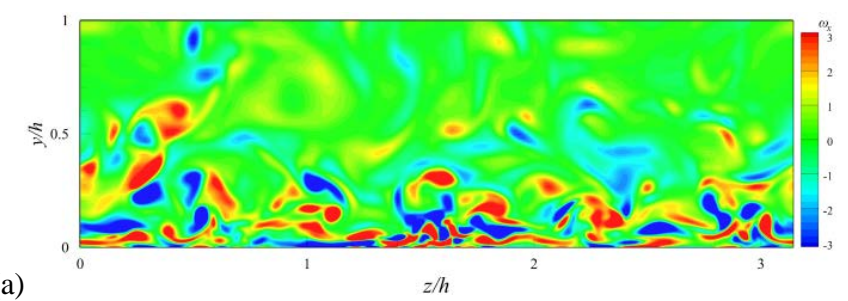

(a)

$z / h$

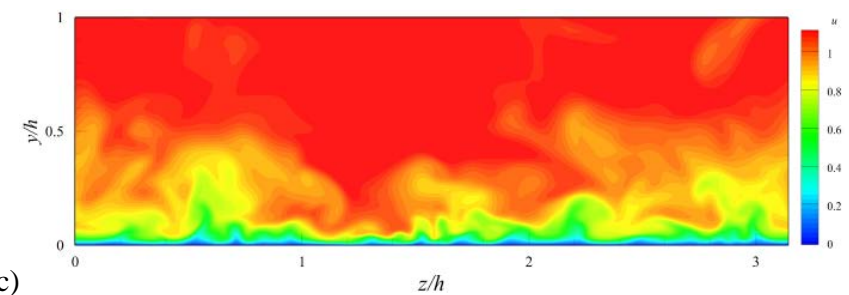

(b)

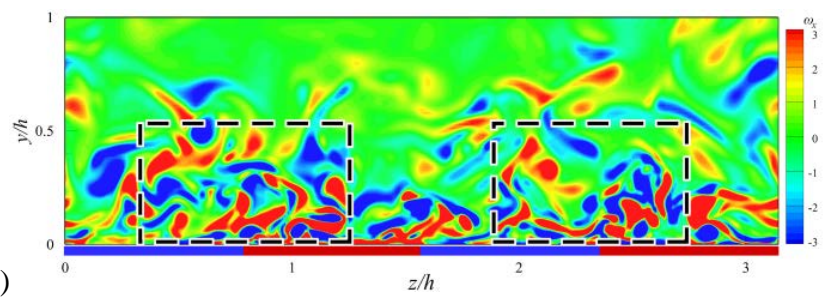

(d)

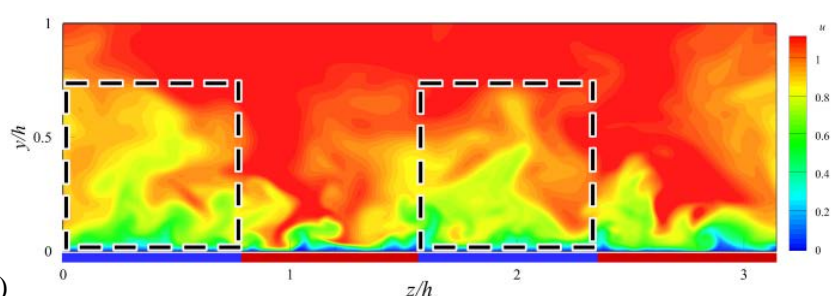

Fig. 14 Instantaneous streamwise vorticity $\omega_{x}(a, b)$ and velocity $u$ in $y$-z plane (c, d) for Case NC (a, c) and Case Nstrip4 (b, d). All the slices are extracted from $x / h=4.8$. The blue and red strips on the lower wall of the channel in (b) and (d) represent the OPC and IPC strips respectively. The dashed line boxes in (b) represent the small-scale vortical flow motions with high values of vorticity. This indicates the presence of LSSVs; the dashed line boxes in (d) stand for the large-scale low-speed region induced by SADS control.

The streamwise velocity fluctuation $u_{\langle y z\rangle}^{\prime}$ extracted from three $x$-z planes at $y^{+}=5,40$ and 200 are presented in Fig. 15. In the near-wall region, a clear difference in the velocity streaks above the OPC and IPC strips is observed (see Fig. 15 (b)) in comparison with the corresponding regions in Case NC as indicated in Fig. 15 (a). At $y^{+}=5$ above the IPC strips, the streak structures are enhanced at a reduced scale with a more twisted shape and greater strength. Above the OPC strips, the opposite trend is observed. The fluid motion is much smoother and it shows fewer and weaker organised structures. At $y^{+}=40$, the boundary between the OPC and IPC strips can not be easily identified, but the difference between the uncontrolled and the controlled cases is still clearly noticeable. The low speed streaks in the controlled case are more chaotic and wider in general, as they are not as compact as those of the uncontrolled case. This might be due to the presence of a vertical shear layer. At $y^{+}$ $=200$, the controlled case presents low speed spots that are more pronounced than for the uncontrolled case, which indicates stronger ejection motions by the controlled case (see, e.g. Fig. 7 (e)).

(a)

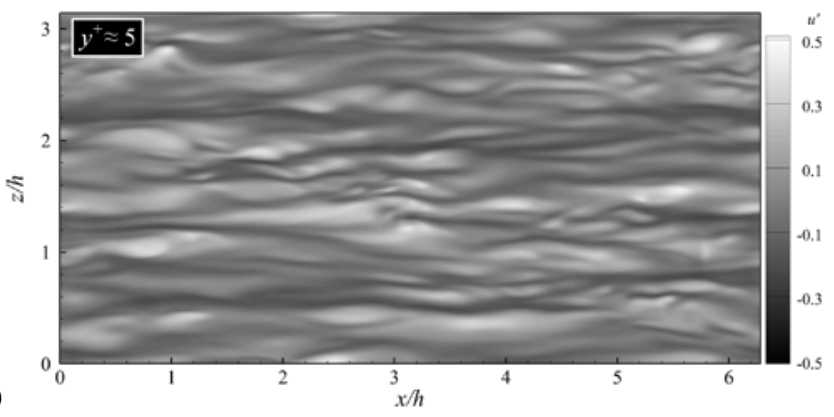

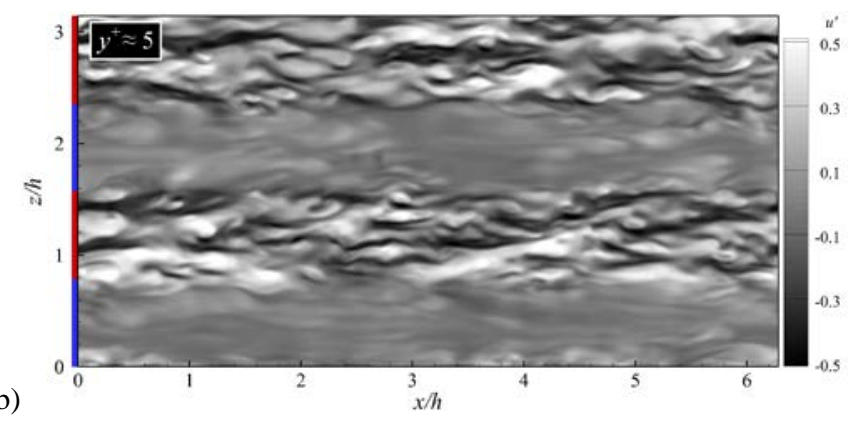


(c)

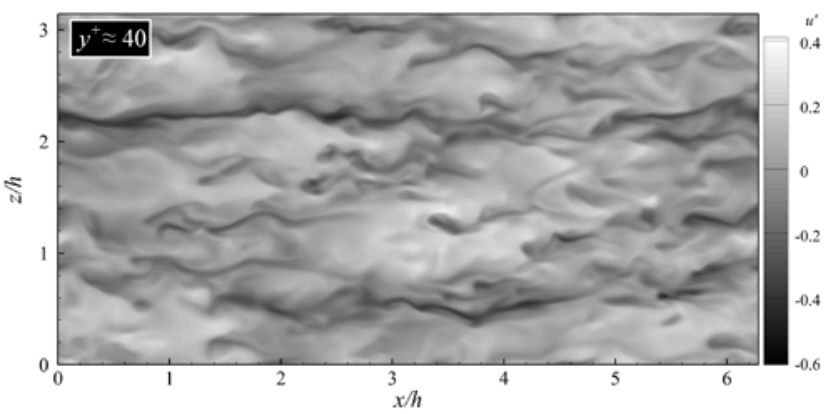

(d)

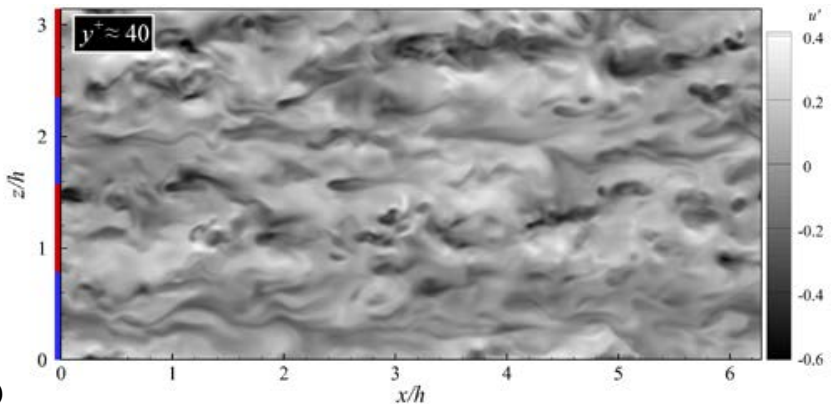

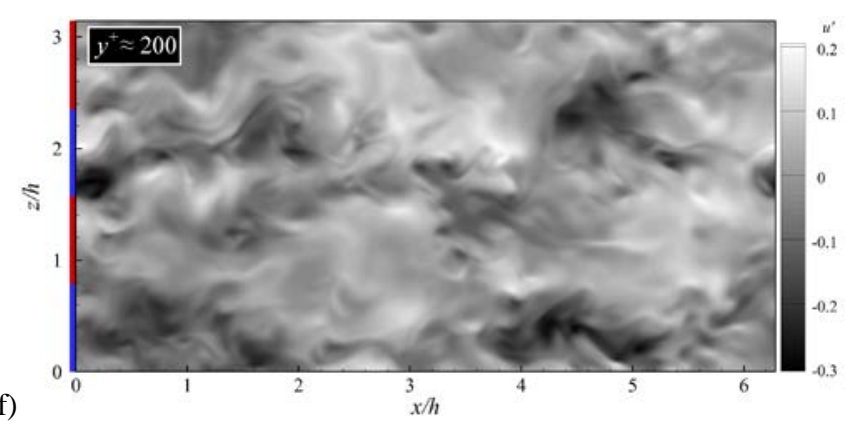

(f) (e)

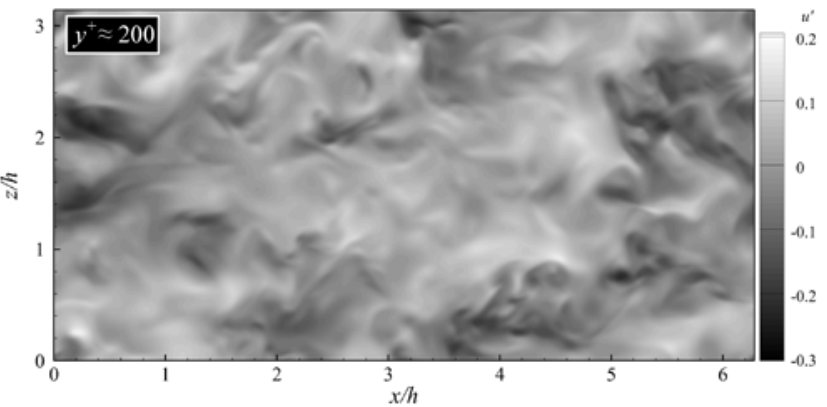

Fig. 15 Streamwise fluctuating velocity streaks for Case NC (left-hand side of the figure) and Case Nstrip4 (right-hand side of the figure). The slices are extracted from the locations of $y^{+}=5,40$ and 200 (top to bottom). The blue and red strips on the right-hand side columns represent the OPC and IPC strips respectively.

\section{CONLUSIONS and PERSPECTIVES}

The turbulent flow in a plane channel whose lower wall is modified by spanwise alternatively distributed out-of-phase and in-phase control using active wall velocity fluctuations has been investigated by DNS at flow conditions of $R e=6,000$ and $M=0.2$. The generation of the large-scale streamwise vortices by such a small-scale control device and their effects on the momentum transport are investigated, as this control strategy could be used to suppress flow separation. Based on this study, the following conclusions are derived:

- Spanwise alternatively distributed OPC/IPC strips have an impact on the flow field, especially in the spanwise direction. The turbulence coherent structures are alternatively suppressed and enhanced by the OPC and IPC strips for all the controlled cases investigated. The skin friction decreases and increases alternatively in the spanwise direction with a vertical shear layer being induced. The mean velocity profiles are also changed along the spanwise direction. Among all the studied cases, the one with 2 pairs of strips has shown the best effect in terms of modification of the turbulence. This corresponds to a strip width of $\Delta z^{+}=264$ (see TABLE 2) for this optimised configuration.

- The analysis shows that the generated LSSV has a statistically steady flow motion and its centre is located at the interfaces between the OPC and IPC strips. Having the sweep side towards the IPC strips, it contributes partly to the generation of high turbulence kinetic energy and Reynolds shear stress in the near-wall region above the IPC strips. As the ejection side moves away from the OPC strips, it generates high turbulence kinetic energy and Reynolds shear stress 
in the central part of the channel above the OPC strips. Therefore, the momentum transport across the channel can be largely enhanced by LSSVs, demonstrating great potential for suppressing flow separation.

- A peak value of the Reynolds shear stress, located at about $0.39 \mathrm{~h}$ from the lower wall of the channel, is exhibited by the case based on 2 pairs of OPC/IPC. This peak value, far from the near-wall region, is induced by the spanwise inhomogeneous variations created by the alternatively distributed strips control in the spanwise direction.

As for the Reynolds number dependence, the effect of Reynolds number on the flow topology transition is conducted by Stroh et al. [47], based on the results from DNS in a turbulent channel flow with alternatively distributing no-slip and free-slip regions on the spanwise direction. They suggest that flow of $R e_{\tau}=120$ and 360 ( $R e_{\tau}=340$ in the present study) reveal a very similar flow topology transition to the one at $R e_{\tau}=180$, which has been studied in detail in their research. Therefore, we might be able to expect that the control method adopted in the present study still works in the flow with different Reynolds number. But further study should be carried to confirm the supposition. The ability to suppress flow separation will be investigated in the future for the boundary layer and backward-facing ramp flows and the underlying mechanism will be studied in detail.

\section{ACKNOWLEDGMENTS}

The project is supported by the Key Subjects of the National Natural Science Foundation of China (51420105008, 11572025 and 51476004), the UK Turbulence Consortium via EPSRC (Grant number EP/L000261/1), and the Department of Research and Advanced Engineering of PSA. The authors also would like to thank EPSRC for the use of ARCHER and also the STFC Hartree Centre for running the simulation and post-processing the data.

Conflicts of Interest: The authors declare that they have no conflict of interest.

\section{REFERENCES}

${ }^{1}$ Kim, J.: Physics and control of wall turbulence for drag reduction. Phil. Trans. R. Soc. A 369, 1396-1411 (2011)

${ }^{2}$ Leschziner, M.A., Choi, H., Choi, K.S.: Flow-control approaches to drag reduction in aerodynamics: progress and prospects.

Phil. Trans. R. Soc. A 369, 1349-1351(2011)

${ }^{3}$ Taylor, H.D.: The elimination of diffuser separation by vortex generators. United Aircraft Corporation Report No. R-4012-3, June 1947

${ }^{4}$ Rao, D.M., Kariya, T.T.: Boundary-layer submerged vortex generators for separation control—an exploratory study. AIAA Paper 88-3546-CP, AIAA/ASME/SIAM/APS 1st National Fluid Dynamics Congress, Cincinnati, OH, July 25-28, 1988 
${ }^{5}$ Lin, J.C., Howard, F.G., Selby, G.V.: Small submerged vortex generators for turbulent flow separation control. J Spacecr Rockets 27, 503-507(1990)

${ }^{6}$ Lin, J.C., Selby, G.V., Howard, F.G.: Exploratory study of vortex-generating devices for turbulent flow separation control. AIAA Paper 91-0042, AIAA 29th Aerospace Sciences Meeting, Reno, NV, January 7-10, 1991

${ }^{7}$ Lin, J.C.: Control of turbulent boundary-layer separation using micro-vortex generators. AIAA Paper 99-3404, 30th AIAA Fluid Dynamics Conference, Norfolk, VA, June 28-July 1, 1999

${ }^{8}$ Jenkins, L., Gorton, S.A., Anders, S.: Flow control device evaluation for an internal flow with an adverse pressure gradient. AIAA Paper 2002-0266, 40th AIAA Aerospace Sciences Meeting and Exhibit, Reno, NV, January 14-17, 2002

${ }^{9}$ Nugroho, B., Hutchins, N., Monty, J.P.: Large-scale spanwise periodicity in a turbulent boundary layer induced by highly ordered and directional surface roughness. Int. J. Heat Fluid Fl. 41, 90-102(2013)

${ }^{10}$ Willingham, D., Anderson, W., Christensen, K. T., Barros, J. M.: Turbulent boundary layer flow over transverse aerodynamic roughness transitions: Induced mixing and flow characterization. Phys. Fluids 26, 25-42(2014)

${ }^{11}$ Mejia-Alvarez, R. Christensen, K.T.: Wall-parallel stereo particle-image velocimetry measurements in the roughness sublayer of turbulent flow overlying highly irregular roughness. Phys. Fluids 25, 115109 (2013)

${ }^{12}$ Mejia-Alvarez, R., Barros, J. M., Christensen, K. T.: Structural attributes of turbulent flow over a complex topography. Coherent Flow Structures at Earth's Surface 25-41 (2013)

${ }^{13}$ Ganapathisubramani, B., Longmire, E. K., Marusic, I.: Characteristics of vortex packets in turbulent boundary layers. J. Fluid Mech. 478, 35-46 (2003)

${ }^{14}$ Tomkins, D., Adrian, R. J.: Spanwise structure and scale growth in turbulent boundary layers. J. Fluid Mech. 490, 3774(2003)

${ }^{15}$ Kline, S.J., Reynolds, W.C., Schraub, F.A., Rundstadler, P.W.: The structure of turbulent boundary layers. J. Fluid Mech. 30, 741-773 (1967)

${ }^{16}$ Robinson, S.K.: Coherent Motions in the Turbulent Boundary Layer. Annu. Rev. Fluid Mech. 23, 601-639(1991)

${ }^{17}$ Schoppa, W., Hussain, F.: Coherent structure generation in near-wall turbulence. J. Fluid Mech. 453, 57-108(2002)

${ }^{18}$ Vermaas, A., Uijttewaal, W.S.J., Hoitink, A.J.F.: Lateral transfer of streamwise momentum caused by a roughness transition across a shallow channel. Water Resources Research 47, 2144-2150(2011)

${ }^{19}$ Mejia-Alvarez, R., Christensen, K.T.: Low-order representations of irregular surface roughness and their impact on a turbulent boundary layer. Phys. Fluids 22, 015106 (2010)

${ }^{20}$ Hinze, J.O.: Secondary currents in wall turbulence. Phys Fluids 10, S122-S125(1967). 
${ }^{21}$ Hinze J.O.: Experimental investigation on secondary currents in the turbulent flow through a straight conduit. Appl Sci Res. 28, 453-465(1973).

${ }^{22}$ Townsend, A.A.: The Structure of Turbulent Shear Flow. Cambridge University Press, London (1956)

${ }^{23}$ Koeltzsch, K., Dinkelacker, A., Grundmann, R.: Flow over convergent and divergent wall riblets. Experiments in Fluids 33, 346-350(2002)

${ }^{24}$ Fang, J., Yao, Y., Li, Z., Lu, L.: Investigation of low-dissipation monotonicity-preserving scheme for direct numerical simulation of compressible turbulent flows. Comput Fluids 104, 55-72(2014)

${ }^{25}$ Fang, J., Yao, Y., Zheltovodov, A.A., Li, Z., Lu, L.: Direct numerical simulation of supersonic turbulent flows around a tandem expansion-compression corner. Phys. Fluids 27, 125104 (2015)

${ }^{26}$ Fang, J., Yao, Y., Zheltovodov A.A., Lu L.: Investigation of Three-Dimensional Shock Wave/Turbulent-Boundary-Layer Interaction Initiated by a Single Fin. AIAA J. 55, 509-523 (2017)

${ }^{27}$ Lele, S.K.: Compact finite difference schemes with spectral-like resolution. J. Comput. Phys. 103, 16-42(1992)

${ }^{28}$ Sandham, N.D., Li, Q., Yee, H.C.: Entropy splitting for high-order numerical simulation of compressible turbulence. J.

Comput. Phys. 178, 307-322(2002)

${ }^{29}$ Gaitonde, D.V., Visbal, M.R.: Pade-Type Higher-Order Boundary Filters for the Navier-Stokes Equations. AIAA J. 38, 2103-2112(2000)

${ }^{30}$ Ottlieb, S. Shu, C.W.: Total variation diminishing Runge-Kutta schemes. Math. Comput. 67, 73-85(1998)

${ }^{31}$ Sagaut, P.: Theoretical background: large-eddy simulation, large-eddy simulation for acoustics. Cambridge University

Press(2007)

${ }^{32}$ Lenormand, E., Sagaut, P., Phuoc, L.T.: Large eddy simulation of subsonic and supersonic channel flow at moderate

Reynolds number. Int. J. Numer. Meth. Fluids 32, 369-406(2000)

${ }^{33}$ Choi, H., Moin, P., Kim, J.: Active turbulence control for drag reduction in wall-bounded flows. J. Fluid Mech. 262, 75110(1994)

${ }^{34}$ Fang, J., Lu, L.: Large eddy simulation of compressible turbulent channel flow with active spanwise wall fluctuations. Modern Physics Letter B 24, 1457-1460(2010)

${ }^{35}$ Moser, R.D., Kim, J., Mansour, N.N.: Direct numerical simulation of turbulent channel flow up to $R e_{\tau}=590$. Phys. Fluids 11, 943-945(1999)

${ }^{36}$ Choi, H., Moin, P., Kim, J.: Direct numerical simulation of turbulent flow over riblets. J. Fluid Mech. 255, 503-539(1993) 
${ }^{37}$ Hansen, K.L., Rostamzadeh, N., Kelso, R.M., Dally, B.B.: Evolution of the streamwise vortices generated between leading edge tubercles. J. Fluid Mech., 788, 730-766(2016)

${ }^{38}$ Kim, J., Moin, P., Moser, R.: Turbulence statistics in fully developed channel flow at low Reynolds number. J. Fluid Mech. 177, 133-166(1987).

${ }^{39}$ Jiménez, J.: Near-wall turbulence. Phys. Fluids, 25, 101302(2013).

${ }^{40}$ Barros, J.M., Christensen, K.T.: Observations of turbulent secondary flows in a rough-wall boundary layer. J. Fluid Mech. 748, R1(2014)

${ }^{41}$ Kevin, K., Monty, J.P., Bai, H.L., Pathikonda, G., Nugroho, B., Barros, J.M., Christensen, K.T., Hutchins, N.: Cross-stream stereoscopic particle image velocimetry of a modified turbulent boundary layer over directional surface pattern. J. Fluid Mech. 813, 412-435(2017).

${ }^{42}$ Hansen, K.L., Rostamzadeh, N., Kelso, R.M., Dally, B.B.: Evolution of the streamwise vortices generated between leading edge tubercles. J. Fluid Mech. 788, 730-766(2016).

${ }^{43}$ Zhou, J., Adrian, R.J., Balachandar, S., Kendall, T. M.: Mechanisms for generating coherent packets of hairpin vortices in channel flow. J. Fluid Mech. 387, 353-396(1999)

${ }^{44}$ Adrian, R.J., Christensen, K.T., Liu, Z.C.: Analysis and interpretation of instantaneous turbulent velocity fields. Exp. Fluids 29, 275-290(2000)

${ }^{45}$ Adrian, R.J.: Hairpin vortex organization in wall turbulence. Phys. Fluids 19, 041301(2007)

${ }^{46}$ Adrian, R.J., Meinhart, C.D., Tomkins, C.D.: Vortex organization in the outer region of the turbulent boundary layer. J. Fluid Mech. 422, 1-54(2000)

${ }^{47}$ Stroh, A., Hasegawa, Y., Kriegseis, J., Frohnapfel B.: Secondary vortices over surfaces with spanwise varying drag. J. Turbul. 17, 1142-1158(2016). 